\title{
A MEASUREMENT OF GRAVITATIONAL LENSING OF THE MICROWAVE BACKGROUND USING SOUTH POLE TELESCOPE DATA
}

\author{
A. van Engelen ${ }^{1}$, R. Keisler ${ }^{2,3}$, O. ZahN ${ }^{4}$, K. A. Aird ${ }^{5}$, B. A. Benson ${ }^{2,6}$, L. E. Bleem ${ }^{2,3}$, J. E. CARlstrom ${ }^{2,3,6,7,8}$, \\ C. L. Chang $^{2,6,8}$, H. M. Cho ${ }^{9}$, T. M. Crawford ${ }^{2,7}$, A. T. Crites $^{2,7}$, T. DE HaAn ${ }^{1}$, M. A. Dobbs ${ }^{1}$, J. Dudley ${ }^{1}$, E. M. George ${ }^{10}$, \\ N. W. Halverson ${ }^{11}$, G. P. Holder ${ }^{1}$, W. L. Holzapfel ${ }^{10}$, S. Hoover ${ }^{2,3}$, Z. Hou ${ }^{12}$, J. D. HrubeS ${ }^{5}$, M. JoY $^{13}$, L. KNOX $^{12}$,

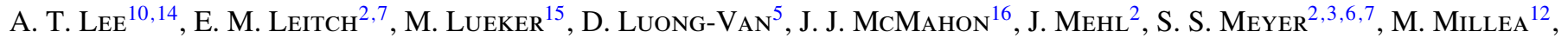 \\ J. J. Mohr ${ }^{17,18,19}$, T. E. Montroy ${ }^{20}$, T. Natoli ${ }^{2,3}$, S. Padin ${ }^{2,7,15}$, T. Plagge ${ }^{2,7}$, C. Pryke ${ }^{21}$, C. L. Reichardt ${ }^{10}$, J. E. Ruhl ${ }^{20}$, \\ J. T. Sayre ${ }^{20}$, K. K. Schaffer ${ }^{2,6,22}$, L. Shaw ${ }^{23}$, E. Shirokoff ${ }^{9}$, H. G. Spieler ${ }^{14}$, Z. Staniszewski ${ }^{20}$, A. A. Stark $^{24}$, \\ K. StORY ${ }^{2,3}$, K. VANDERLinde ${ }^{1}$, J. D. Vieira ${ }^{15}$, AND R. WilliamsON ${ }^{2,7}$ \\ ${ }^{1}$ Department of Physics, McGill University, 3600 Rue University, Montreal, Quebec H3A 2T8, Canada \\ ${ }^{2}$ Kavli Institute for Cosmological Physics, University of Chicago, 5640 South Ellis Avenue, Chicago, IL 60637, USA \\ ${ }^{3}$ Department of Physics, University of Chicago, 5640 South Ellis Avenue, Chicago, IL 60637, USA \\ ${ }^{4}$ Berkeley Center for Cosmological Physics, Department of Physics, University of California, \\ and Lawrence Berkeley National Labs, Berkeley, CA 94720, USA \\ ${ }^{5}$ University of Chicago, 5640 South Ellis Avenue, Chicago, IL 60637, USA \\ ${ }^{6}$ Enrico Fermi Institute, University of Chicago, 5640 South Ellis Avenue, Chicago, IL 60637, USA \\ ${ }^{7}$ Department of Astronomy and Astrophysics, University of Chicago, 5640 South Ellis Avenue, Chicago, IL 60637, USA \\ ${ }^{8}$ Argonne National Laboratory, 9700 S. Cass Avenue, Argonne, IL 60439, USA \\ ${ }^{9}$ NIST Quantum Devices Group, 325 Broadway Mailcode 817.03, Boulder, CO 80305, USA \\ ${ }^{10}$ Department of Physics, University of California, Berkeley, CA 94720, USA \\ ${ }^{11}$ Department of Astrophysical and Planetary Sciences and Department of Physics, University of Colorado, Boulder, CO 80309, USA \\ ${ }_{12}$ Department of Physics, University of California, One Shields Avenue, Davis, CA 95616, USA \\ ${ }^{13}$ Department of Space Science, VP62, NASA Marshall Space Flight Center, Huntsville, AL 35812, USA \\ ${ }^{14}$ Physics Division, Lawrence Berkeley National Laboratory, Berkeley, CA 94720, USA \\ ${ }^{15}$ Division of Physics, Mathematics, and Astronomy, California Institute of Technology, MS 249-17, \\ 1216 East California Boulevard, Pasadena, CA 91125, USA \\ ${ }^{16}$ Department of Physics, University of Michigan, 450 Church Street, Ann Arbor, MI 48109, USA \\ ${ }^{17}$ Department of Physics, Ludwig-Maximilians-Universität, Scheinerstr. 1, D-81679 München, Germany \\ ${ }^{18}$ Excellence Cluster Universe, Boltzmannstr. 2, D-85748 Garching, Germany \\ ${ }^{19}$ Max-Planck-Institut für extraterrestrische Physik, Giessenbachstr. 1, D-85748 Garching, Germany \\ ${ }^{20}$ Physics Department, Center for Education and Research in Cosmology and Astrophysics, \\ Case Western Reserve University, Cleveland, OH 44106, USA \\ ${ }^{21}$ Department of Physics, University of Minnesota, 116 Church Street S.E., Minneapolis, MN 55455, USA \\ ${ }^{22}$ Liberal Arts Department, School of the Art Institute of Chicago, 112 S Michigan Avenue, Chicago, IL 60603, USA \\ ${ }^{23}$ Department of Physics, Yale University, P.O. Box 208210, New Haven, CT 06520-8120, USA \\ ${ }^{24}$ Harvard-Smithsonian Center for Astrophysics, 60 Garden Street, Cambridge, MA 02138, USA \\ Received 2012 February 3; accepted 2012 June 25; published 2012 August 23
}

\begin{abstract}
We use South Pole Telescope data from 2008 and 2009 to detect the non-Gaussian signature in the cosmic microwave background (CMB) produced by gravitational lensing and to measure the power spectrum of the projected gravitational potential. We constrain the ratio of the measured amplitude of the lensing signal to that expected in a fiducial $\Lambda \mathrm{CDM}$ cosmological model to be $0.86 \pm 0.16$, with no lensing disfavored at $6.3 \sigma$. Marginalizing over $\Lambda \mathrm{CDM}$ cosmological models allowed by the Wilkinson Microwave Anisotropy Probe (WMAP7) results in a measurement of $A_{\text {lens }}=0.90 \pm 0.19$, indicating that the amplitude of matter fluctuations over the redshift range $0.5 \lesssim z \lesssim 5$ probed by CMB lensing is in good agreement with predictions. We present the results of several consistency checks. These include a clear detection of the lensing signature in CMB maps filtered to have no overlap in Fourier space, as well as a "curl" diagnostic that is consistent with the signal expected for $\Lambda \mathrm{CDM}$. We perform a detailed study of bias in the measurement due to noise, foregrounds, and other effects and determine that these contributions are relatively small compared to the statistical uncertainty in the measurement. We combine this lensing measurement with results from WMAP7 to improve constraints on cosmological parameters when compared to those from WMAP7 alone: we find a factor of 3.9 improvement in the measurement of the spatial curvature of the universe, $\Omega_{k}=-0.0014 \pm 0.0172$; a $10 \%$ improvement in the amplitude of matter fluctuations within $\Lambda \mathrm{CDM}, \sigma_{8}=0.810 \pm 0.026$; and a $5 \%$ improvement in the dark energy equation of state, $w=-1.04 \pm 0.40$. When compared with the measurement of $w$ provided by the combination of WMAP7 and external constraints on the Hubble parameter, the addition of the lensing data improves the measurement of $w$ by $15 \%$ to give $w=-1.087 \pm 0.096$.
\end{abstract}

Key words: cosmic background radiation - cosmological parameters - cosmology: observations - gravitational lensing: weak - large-scale structure of universe

Online-only material: color figures 


\section{INTRODUCTION}

Measurements of the cosmic microwave background (CMB) have allowed us to infer much about the universe during the epoch of recombination (most recently Jarosik et al. 2011; Das et al. 2011a; Keisler et al. 2011). Over the last decade, measurements of interactions between the CMB and structures at lower redshifts have been used to constrain cosmology. These include the measurement of the effects of reionization on the CMB at $z \sim 10$ (e.g., Kogut et al. 2003; Komatsu et al. 2011; Zahn et al. 2011); the detection of the integrated Sachs-Wolfe effect from the onset of dark energy domination at $z \sim 1$ (e.g., Fosalba et al. 2003; Padmanabhan et al. 2005); and large surveys using the Sunyaev-Zel'dovich (SZ) effect (Sunyaev \& Zel'dovich 1972) to measure the growth of structure and the present-day amplitude of matter fluctuations (e.g., Vanderlinde et al. 2010; Sehgal et al. 2011; Dunkley et al. 2011; Reichardt et al. 2011; Benson et al. 2011).

The gravitational lensing of the CMB is another longpromised source of information on the post-recombination universe (e.g., Blanchard \& Schneider 1987; Cole \& Kaiser 1989; Seljak 1996; for a review see Lewis \& Challinor 2006). Lensing affects the CMB in two ways: it smooths the CMB temperature power spectrum, and it correlates initially independent modes. A measurement of this latter effect can be obtained, for instance, with the optimal quadratic estimator technique of Seljak \& Zaldarriaga (1999), Hu (2001b), and Okamoto \& Hu (2003), giving a reconstruction of the projected gravitational potential (Bernardeau 1997; Seljak \& Zaldarriaga 1999; Zaldarriaga \& Seljak 1999; Hu 2001b). Most of the weight in the projection comes from high redshifts, with the maximum at $z \sim 2$. $\mathrm{CMB}$ lensing measurements can thus probe the physics affecting structure formation at high redshift, including the sum of the neutrino masses (Kaplinghat et al. 2003; Lesgourgues et al. 2006). In addition, the gravitational potential can be probed on very large scales, leading to constraints on curvature, dark energy, and modified gravity models (Smith et al. 2006; Calabrese et al. 2009).

Until recently, CMB observations had insufficient sensitivity and angular resolution to detect lensing with the CMB alone. Smith et al. (2007) instead performed a cross-correlation between a reconstruction of the nearly full-sky CMB lensing field, obtained with the third-year Wilkinson Microwave Anisotropy Probe (WMAP) data, and the distribution of radio galaxies found in the NRAO VLA Sky Survey (NVSS). This resulted in a detection of the signature of CMB lensing at 3.4 $\sigma$. Similarly, Hirata et al. (2008) found a $2.5 \sigma$ detection of cross-correlation between their lensing map, obtained with the third-year WMAP release using a slightly different estimator, and data from both the Sloan Digital Sky Survey and NVSS.

Experiments with smaller beam sizes and lower noise levels have enabled lensing detections using the CMB alone. The Arcminute Cosmology Bolometer Array Receiver (ACBAR; Reichardt et al. 2009), Atacama Cosmology Telescope (ACT; Dunkley et al. 2011), and South Pole Telescope (SPT; Keisler et al. 2011) teams have found a preference for lensing in the small-scale CMB temperature power spectrum at significances of $\sim 2 \sigma, 2.8 \sigma$, and $5 \sigma$, respectively. Also, although the WMAP satellite is not optimized for the study of the CMB power spectrum damping tail, which is most affected by lensing, the maps in the seventh-year WMAP release (Jarosik et al. 2011) contain low enough noise that evidence for lensing using a novel kurtosis estimator was claimed at $2 \sigma$ by Smidt et al.
(2011). However, Feng et al. (2012) recently applied the optimal quadratic estimator of $\mathrm{Hu}(2001 \mathrm{~b})$ and Okamoto \& $\mathrm{Hu}$ (2003) to the WMAP7 maps, finding no significant signal. The first clear detection of the power spectrum of the CMB lensing potential was obtained by Das et al. (2011b), who used the quadratic estimator approach with ACT maps to obtain a $4 \sigma$ detection.

Here, we perform quadratic lensing reconstruction and present a detection of the lensing power spectrum from $590 \mathrm{deg}^{2}$ of CMB sky observed by the SPT in 2008 and 2009. This sky area is approximately twice that used by Das et al. (2011b), and is observed with $\sim 25 \%$ lower noise. The paper is structured as follows. In Section 2, we review lensing of the CMB. In Section 3, we briefly review the SPT data set, noting that we use the maps which were generated for the CMB power spectrum analysis of Keisler et al. (2011, hereafter K11). In Section 4, we detail our application of the quadratic estimator technique to the SPT maps. In Section 5, we estimate the impact of foregrounds and other systematic effects in the data, and show that they can be controlled for the current level of precision. In Section 6, we present the quantitative results, including a measurement of the amplitude of the lensing power spectrum relative to theoretical expectations and constraints on cosmological parameters. We conclude in Section 7.

\section{CMB LENSING}

As CMB photons travel toward us, their paths are slightly deflected by fluctuations in the intervening matter density. Since the fluctuations are mostly in the linear regime on large scales, each deflection is small, and we can consider the total deflection for a given observation direction $\hat{\mathbf{n}}$ as a sum of deflections along the line of sight. The projected potential for lensing $\phi(\hat{\mathbf{n}})$ is then given by

$$
\phi(\hat{\mathbf{n}})=-2 \int_{0}^{\chi_{\mathrm{CMB}}} d \chi \frac{f_{K}\left(\chi_{\mathrm{CMB}}-\chi\right)}{f_{K}\left(\chi_{\mathrm{CMB}}\right) f_{K}(\chi)} \Phi(\chi \hat{\mathbf{n}}, \chi),
$$

where $\Phi(\mathbf{r}, \eta)$ is the three-dimensional gravitational potential at position $\mathbf{r}$ and conformal look-back time $\eta$, both measured with us at the origin, $\chi$ is the comoving distance along the line of sight, $\chi_{\mathrm{CMB}} \simeq 14 \mathrm{Gpc}$ is the comoving distance to the $\mathrm{CMB}$, and $f_{K}(\chi)$ is the comoving angular diameter distance, with $f_{K}(\chi)=\chi$ in a spatially flat universe. Lensing shifts the unlensed CMB temperature $T^{U}(\hat{\mathbf{n}})$ at a sky position $\hat{\mathbf{n}}$ by the gradient of this lensing potential, resulting in an observed $\mathrm{CMB}$ temperature

$$
\begin{aligned}
T(\hat{\mathbf{n}}) & =T^{U}(\hat{\mathbf{n}}+\nabla \phi(\hat{\mathbf{n}})) \\
& =T^{U}(\hat{\mathbf{n}})+\nabla T^{U}(\hat{\mathbf{n}}) \cdot \nabla \phi(\hat{\mathbf{n}})+\ldots
\end{aligned}
$$

The statistics of the Gaussian, unlensed temperature field are determined purely by the unlensed CMB power spectrum $C_{l}^{U}$ according to

$$
\left\langle T^{U}\left(\mathbf{l}_{1}\right) T^{U}\left(\mathbf{l}_{2}\right)\right\rangle=(2 \pi)^{2} \delta\left(\mathbf{l}_{1}+\mathbf{l}_{2}\right) C_{l_{1}}^{U},
$$

where we use the flat-sky Fourier convention

$$
T(\mathbf{l})=\int d^{2} \hat{\mathbf{n}} T(\hat{\mathbf{n}}) e^{-i \mathbf{l} \cdot \hat{\mathbf{n}}},
$$

and apply the high- $l$ limit in which the all-sky power spectrum $C_{l}$ becomes equivalent to its flat-sky Fourier analog. 
When the CMB is lensed, the coupling between the CMB gradient and the $\phi$ gradient in Equation (2) leads to an offdiagonal correlation between CMB multipole moments of

$$
\begin{aligned}
\left\langle T\left(\mathbf{l}_{1}\right) T\left(\mathbf{l}_{2}\right)\right\rangle & =\mathbf{L} \cdot\left(\mathbf{l}_{1} C_{l_{1}}^{U}+\mathbf{l}_{2} C_{l_{2}}^{U}\right) \phi(\mathbf{L}) \\
& \equiv f\left(\mathbf{l}_{1}, \mathbf{l}_{2}\right) \phi(\mathbf{L}),
\end{aligned}
$$

at linear order in $\phi$. Here, $\mathbf{L}=\mathbf{l}_{1}+\mathbf{l}_{2}$, and we have assumed $\mathbf{l}_{1} \neq-\mathbf{l}_{2} \cdot{ }^{25}$

A quadratic estimator takes advantage of this off-diagonal coupling by averaging over products of pairs of observed CMB modes $T\left(\mathbf{l}_{1}\right)$ and $T\left(\mathbf{l}_{2}\right)$ that satisfy $\mathbf{l}_{1}+\mathbf{l}_{2}=\mathbf{L} \neq 0$ to reconstruct a mode $\phi(\mathbf{L})$. This is distinct from lensing detections using the CMB temperature power spectrum (Calabrese et al. 2008; Reichardt et al. 2009; Das et al. 2011a; Keisler et al. 2011), which probe the effects of lensing on "on-diagonal" CMB modes with $\mathbf{l}_{1}+\mathbf{l}_{2}=0$.

Although the typical deflection angle is small, $|\nabla \phi|_{\text {RMS }} \simeq$ $2 ! 4$, the lensing deflection field is coherent across several degrees on the sky. The mode coupling is thus strongest for pairs of modes in the CMB map with small vectorial separation, of magnitude $L \lesssim 1000$. Additionally, the signal is most significant on scales at which the CMB temperature power spectrum is a steep function of $l$ (e.g., Zahn \& Zaldarriaga 2006; Bucher et al. 2012). This condition is met on small scales (high $l$ ), where the primary CMB fluctuations are exponentially damped due to photon diffusion effects during recombination. Thus, an ideal lensing estimate will search for nonzero coupling between pairs of $l>1000 \mathrm{CMB}$ modes, separated by $\mathbf{L}$ of several hundred. Data from the current generation of CMB temperature experiments, namely, SPT, ACT, and the Planck Surveyor, can be used to resolve the fluctuations in the damping tail region of the CMB temperature power spectrum. These data are thus well suited for measuring the lensing signal on degree scales, using only the measured fluctuations on scales of several arcminutes.

\section{THE SOUTH POLE TELESCOPE}

The SPT is an off-axis Gregorian telescope with a $10 \mathrm{~m}$ diameter primary mirror located at the South Pole. The receiver is equipped with 960 horn-coupled spiderweb bolometers with superconducting transition-edge sensors. The detectors are divided between three frequency bands centered at 95, 150, and $220 \mathrm{GHz}$. The telescope and receiver are discussed in more detail in Ruhl et al. (2004), Padin et al. (2008), and Carlstrom et al. (2011).

\subsection{Survey and Fields}

The SPT-SZ survey is a multi-year observation program with the principal goals of using the SZ effect to produce a nearly mass-limited sample of galaxy clusters for cosmological studies, e.g., for measuring the growth of structure to constrain the dark energy equation of state (Staniszewski et al. 2009; Vanderlinde et al. 2010; Foley et al. 2011; Williamson et al. 2011) and to measure the power spectrum of the millimeter-wave sky on small angular scales (Lueker et al. 2010; Hall et al. 2010; Shirokoff et al. 2011; K11; Reichardt et al. 2011).

In this work, we use only the $150 \mathrm{GHz}$ due to the lower noise, of approximately $18 \mu \mathrm{K}$ arcmin. ${ }^{26}$ We use the two fields

\footnotetext{
25 Throughout the paper, we use capital $\mathbf{L}$ to refer to the argument of the lensing field, and lowercase $\mathbf{I}$ to refer to the argument of the CMB temperature field.

26 Throughout this work, map signal and noise amplitudes are expressed in units of $\mathrm{K}-\mathrm{CMB}$, expressing deviations from the average measured intensity as equivalent temperature fluctuations in the CMB.
}

observed by the SPT in 2008, which total $197 \mathrm{deg}^{2}$, and two of the fields observed in 2009, which total $393 \mathrm{deg}^{2}$. These four fields correspond to the fields marked RA5H30DEC-55, RA23H30DEC-55, RA3H30DEC-60, and RA21HDEC-60 in Table 1 of K11.

\subsection{Mapmaking from Time Streams}

The SPT maps used in this analysis are identical to those used by K11. The processing used to go from time-ordered data (TOD) to maps is described in more detail in that work, and we summarize the main points here. First, the raw $100 \mathrm{~Hz}$ TOD are low-pass filtered at $7.5 \mathrm{~Hz}$ and resampled at $16.7 \mathrm{~Hz}$. Next, the TOD are bandpass filtered by applying a second low-pass filter at $5 \mathrm{~Hz}$ and by removing a Legendre polynomial from each scan across the field. Approximately, 1.5 degrees of freedom are removed per degree on the sky. Finally, we subtract the mean signal across each detector module ${ }^{27}$ at each time sample. This spatial high-pass filter removes atmospheric noise that is correlated among detectors.

The filtered TOD, in conjunction with the pointing information, are projected onto two-dimensional maps using the oblique Lambert equal-area azimuthal projection (Snyder 1987) with pixels of size $1^{\prime}$.

All SPT temperature power spectrum analyses are performed using cross-power estimates between maps of disjoint observations of each SPT field, with each observation consisting of several hours of TOD. By contrast, in the lensing analysis, we use only the season-averaged map for each field.

\subsection{Source Removal}

To remove bright sources from the maps, we center a square mask on each source and "paint in" CMB fluctuations using interpolation.

We first derive a source list for masking extremely bright sources and galaxy clusters using SPT catalogs (compiled using maps that were processed slightly differently and with smaller pixels). A $12^{\prime} \times 12^{\prime}$ mask is applied to positive sources brighter than $40 \sigma$ and galaxy clusters brighter than $20 \sigma$. The source densities of extremely bright sources and galaxy clusters are $\sim 0.2$ and $0.01 \mathrm{deg}^{-2}$, respectively. We discuss the dependence on masking levels in Section 5.

Next, fainter sources are identified by applying a matched filter to the maps used for the lensing analysis (which are not optimized for point-source detection) and selecting all sources above $6 \sigma$. These sources are removed by masking the surrounding $8^{\prime} \times 8^{\prime}$ region. The effective flux cut is approximately $10 \mathrm{mJy}$, with a typical source density of 0.5 sources $\mathrm{deg}^{-2}$.

An estimate of the CMB fluctuations in the masked region is then determined in a $16^{\prime}$ square surrounding each source region, where a Gaussian random field is assumed. The covariance matrix $\mathbf{C}$ between pixels in the $16^{\prime}$ square is calculated from a large number of $16^{\prime}$ square regions of SPT maps. A new matrix $\mathbf{C}^{\prime}$ is constructed by setting the diagonal elements corresponding to the masked pixels to large values. Finally, the interpolated map is estimated as $T_{\text {est }}=\mathbf{C C}^{\prime-1} T_{\text {map }}$, where $T_{\text {map }}$ is the original map. This procedure is a variant of Wiener filtering (e.g., Knox et al. 1998). It is analogous to maximum likelihood mapmaking for a small subregion that has high noise embedded in a larger well-measured map that has known large-scale correlations. It

\footnotetext{
27 The SPT array consists of six wedge-shaped bolometer modules, each with 160 detectors. Each wedge is configured with a set of filters that determine its observing frequency $(95,150$, or $220 \mathrm{GHz})$.
} 
is also similar to a constrained realization in the masked region (e.g., Hoffman \& Ribak 1991), but differs in that there is no random noise added on small scales.

The fractional residual power from a point source after this masking procedure is applied is less than $6 \times 10^{-4}$; the total fraction of sky area masked is $\sim 1 \%$. We perform the same procedure on the simulated observations described below to include their impact on the final results.

\subsection{Beam}

The beam shape is measured using a combination of observations of planets and bright point sources. The central beam is approximately Gaussian and is measured using the five brightest point sources in the fields observed by SPT in 2008 and 2009. This approach naturally takes into account the effective beam enlargement due to random errors in the pointing reconstruction. The outer beam, which accounts for roughly $15 \%$ of the total beam solid angle, is measured with planet observations. The SPT beams are described in more detail in K11. We discuss the impact of the beam uncertainties on the lensing results in Section 5.4 .

\section{LENSING ANALYSIS}

In this section, we describe our procedure for measuring the lensing signal. We discuss our method for making lensing maps, give an overview of the detailed end-to-end simulations that play a central role in the analysis, and describe the methods for characterizing the lensing power spectrum.

\subsection{Estimating Lensing Maps from CMB Maps}

Several methods have been proposed to detect the lensing signature in $\mathrm{CMB}$ maps. The most well-studied estimators reconstruct lensing using the coupled nature of CMB modes in the Fourier domain (Bernardeau 1997; Zaldarriaga 2000), which can be optimized with particular choices of filters (Hu 2001a, 2001b; Hu \& Okamoto 2002; Okamoto \& Hu 2003). Pixelspace versions of these estimators have also been formulated (Bucher et al. 2012; Carvalho \& Moodley 2010). Alternative approaches involve formulating and maximizing a lensing likelihood function in pixel space (Hirata \& Seljak 2003a, 2003b; Anderes et al. 2011). However, for temperature-only data with current noise levels, these maximum likelihood approaches are not expected to lead to appreciable gains in signal-to-noise ratio. Therefore, in this work, we use the quadratic formulation to isolate the signature of lensing in the four-point function in the Fourier domain. The same approach was taken in previous detections (Smith et al. 2007; Hirata et al. 2008; Das et al. 2011b).

As shown by $\mathrm{Hu}$ (2001b), the quadratic combination that maximizes the lensing measurement signal to noise is the temperature-weighted gradient, in which one multiplies a filtered CMB gradient with a high-pass filtered CMB map. The filters are chosen to weight the observed CMB according to the inverse variance, and to select for the mode coupling in Equation (5). The gradient-filtered map takes the form (Hu 2001a, 2001b)

$$
\mathbf{G}(\hat{\mathbf{n}})=\int \frac{d^{2} \mathbf{l}}{(2 \pi)^{2}} \frac{C_{l}^{U}}{C_{\mathbf{l}}^{t}} i \mathbf{l} T(\mathbf{l}) e^{i \mathbf{l} \cdot \hat{\mathbf{n}}},
$$

and the high-pass-filtered map takes the form

$$
W(\hat{\mathbf{n}})=\int \frac{d^{2} \mathbf{l}}{(2 \pi)^{2}} \frac{1}{C_{\mathbf{l}}^{t}} T(\mathbf{l}) e^{i \mathbf{l} \cdot \hat{\mathbf{n}}} .
$$

Here, $C_{l}^{U}$ denotes the unlensed CMB power spectrum and $C_{1}^{t}=C_{l}^{L}+C_{1}^{N}+C_{l}^{F}$ denotes the total power in the observed CMB map. The components of this total power include the lensed CMB temperature power $C_{l}^{L}$, the noise power $C_{1}^{N}$, and the power spectrum of the foregrounds $C_{l}^{F}$. We denote the argument of the noise power with a vector, $\mathbf{l}$, due to its anisotropic nature as described below. After forming the real-space product $\mathbf{G}(\hat{\mathbf{n}}) W(\hat{\mathbf{n}})$, one takes the filtered divergence of the result. This leads to an estimate for the scalar lensing deflection field $d(\mathbf{L})$,

$$
\hat{d}(\mathbf{L})=-\frac{A_{\mathbf{L}}}{L} \int d^{2} \hat{\mathbf{n}} \nabla \cdot(\mathbf{G}(\hat{\mathbf{n}}) W(\hat{\mathbf{n}})) e^{-i \mathbf{L} \cdot \hat{\mathbf{n}}},
$$

where $A_{\mathbf{L}}$ is the normalization. The deflection field is related to the lensing potential through $d(\mathbf{L})=L \phi(\mathbf{L})$. Expressed in Fourier space, the estimator is

$$
\hat{d}(\mathbf{L})=\frac{A_{\mathbf{L}}}{L} \int \frac{d^{2} \mathbf{l}_{1}}{(2 \pi)^{2}} F\left(\mathbf{l}_{1}, \mathbf{L}-\mathbf{l}_{1}\right) T\left(\mathbf{l}_{1}\right) T\left(\mathbf{L}-\mathbf{l}_{1}\right),
$$

where the filter function

$$
F\left(\mathbf{l}_{1}, \mathbf{l}_{2}\right)=\frac{f\left(\mathbf{l}_{1}, \mathbf{l}_{2}\right)}{2 C_{\mathbf{l}_{1}}^{t} C_{\mathbf{l}_{2}}^{t}} .
$$

The factor $A_{\mathbf{L}}$ is chosen to normalize the estimate such that to linear order in $\phi$

$$
\langle\hat{d}(\mathbf{L})\rangle_{\mathrm{CMB}}=L \phi(\mathbf{L})
$$

according to Equation (5). The subscript "CMB" on the expectation value indicates that the ensemble average is taken over a set of CMB realizations all lensed by the same $\phi$ field. The function $A_{\mathbf{L}}$ is given, in the absence of data windowing, by

$$
A_{\mathbf{L}}=\left(\frac{1}{L^{2}} \int \frac{d^{2} \mathbf{l}_{1}}{(2 \pi)^{2}} \frac{\left(\mathbf{L} \cdot \mathbf{l}_{1} C_{l_{1}}^{U}+\mathbf{L} \cdot \mathbf{l}_{2} C_{l_{2}}^{U}\right)^{2}}{2 C_{\mathbf{l}_{1}}^{t} C_{\mathbf{I}_{2}}^{t}}\right)^{-1}
$$

This function is also the noise in the lensing estimate ( $\mathrm{Hu} 2001 \mathrm{~b}$; Kesden et al. 2003).

In our simulation-based approach, detailed below, the results of the lensing estimate are not sensitive to the exact settings for the CMB power spectra in the filter; a mismatch between the assumed and exact power will lead to a small loss in optimality, but no bias. We obtain the total CMB power in the denominator, $C_{1}^{t}$, assuming contributions from a lensed WMAP7 best-fit CMB power spectrum (Komatsu et al. 2011); power from uncorrelated point sources of $C_{l} \equiv 7 \times 10^{-6} \mu \mathrm{K}^{2}$; a flatbandpower component with $l^{2} C_{l} / 2 \pi \equiv 10 \mu \mathrm{K}^{2}$ (designed to capture the combination of the power spectra of the thermal SZ effect, the kinetic SZ effect, and clustered dusty galaxies); and finally, a term due to instrumental noise. The two-dimensional noise power spectra of the maps, $C_{\mathbf{l}}^{N}$, are calculated directly from ensembles of SPT difference maps. We obtain pseudoindependent SPT noise realizations, containing little response to any on-sky signal, by flipping the signs of half of the several hundred observations of each field and then performing a co-add.

Due to the SPT observing strategy, in which the telescope scans in azimuth between steps in elevation, the noise power in the maps, $C_{1}^{N}$, is anisotropic. In particular, the noise is substantially larger at low values of $l_{x}$, the Fourier conjugate 


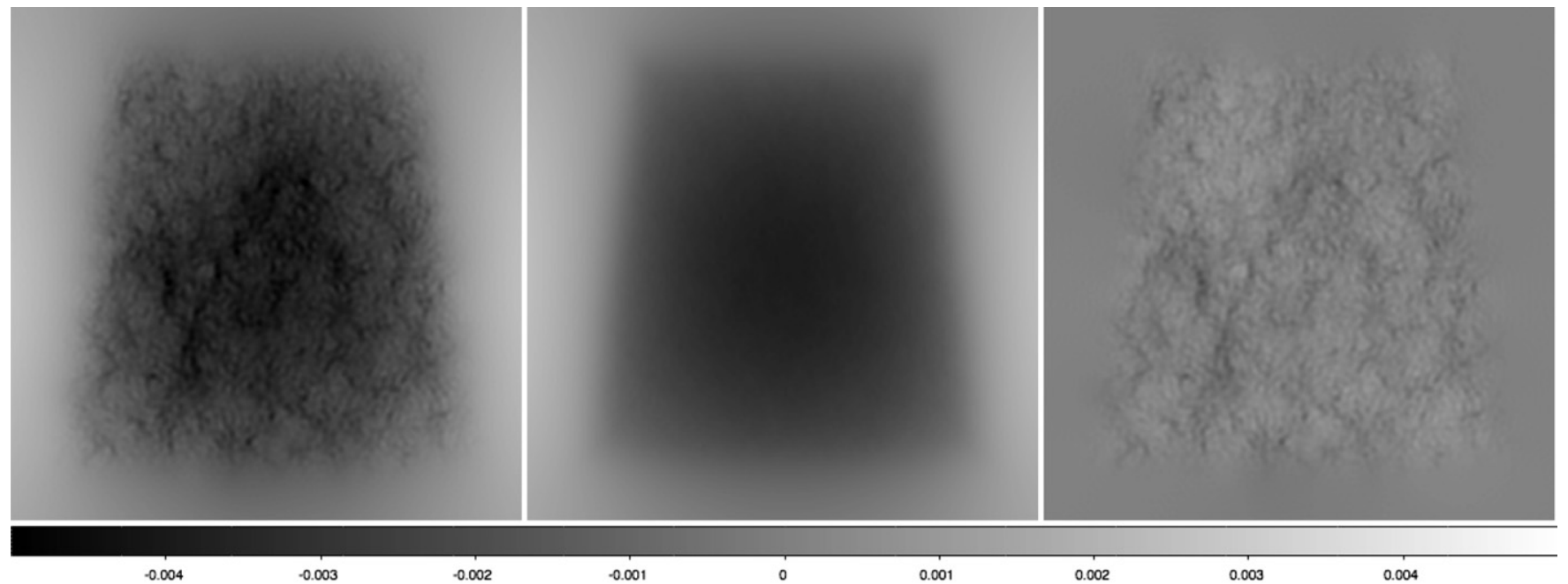

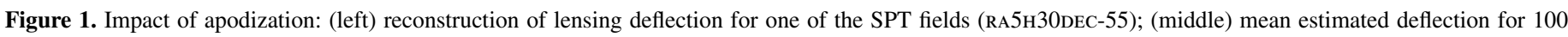

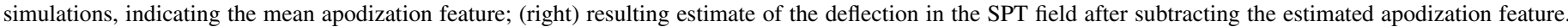
All maps have the same gray scale $( \pm 0.005)$.

to the scan direction. ${ }^{28}$ The lensing estimation procedure, described above, naturally down-weights these modes.

For the given map noise levels of roughly $18 \mu \mathrm{K}-$ arcmin, the lensing signal is concentrated in the annular range of CMB temperature multipoles $1200 \lesssim l \lesssim 3000$. We apply an azimuthally symmetric bandpass filter to isolate these modes. Isolating the temperature modes $T(\mathbf{l})$ within this annulus is effectively equivalent to setting the denominator of the filter in Equation (10), $C_{1}^{t}$, to be infinite outside this annulus.

The instrumental time stream filtering discussed in Section 3.2 leads to a signal transfer function which must be carefully evaluated. This is particularly true at low values of $l_{x}$, which are more aggressively filtered. Although this transfer function was characterized down to low values of $l_{x}$ in $\mathrm{K} 11$, with the lowest reported bin at $l=650$, in this work we do not consider $\mathrm{CMB}$ modes with $l_{x}<1000$ for simplicity. We estimate that a reduction of this cut to lower $l_{x}$ would increase the total lensing detection significance by up to $\sim 10 \%$.

Together, the anisotropic noise and $l_{x}$ filtering lead to an anisotropic function $A_{\mathbf{L}}$, with higher amplitude in the $L_{y^{-}}$ direction than in the $L_{x}$-direction by a factor of $\sim 4$.

\subsubsection{Curl Test}

As described in the previous section, the lensing estimate is derived by taking the divergence of a vector map, $\mathbf{G}(\hat{\mathbf{n}}) W(\hat{\mathbf{n}})$. This estimate is constructed for a field which has a gradient component, but no curl, as expected for the lensing deflection field. By taking the curl instead, we can construct an estimator that closely resembles the lensing estimator, but is optimized for curl-like sources (Cooray et al. 2005). The estimate is formulated as

$$
\hat{c}(\mathbf{L})=-\frac{A_{\mathbf{L}}^{c}}{L} \int d^{2} \hat{\mathbf{n}} \nabla \star(\mathbf{G}(\hat{\mathbf{n}}) W(\hat{\mathbf{n}})) e^{-i \mathbf{L} \cdot \hat{\mathbf{n}}},
$$

with the factor

$$
A_{\mathbf{L}}^{c}=\left(\frac{1}{L^{2}} \int \frac{d^{2} \mathbf{l}_{1}}{(2 \pi)^{2}} \frac{\left(\mathbf{L} \star \mathbf{l}_{1} C_{l_{1}}^{U}+\mathbf{L} \star \mathbf{l}_{2} C_{l_{2}}^{U}\right)^{2}}{2 C_{\mathbf{I}_{1}}^{t} C_{\mathbf{l}_{2}}^{t}}\right)^{-1} .
$$

\footnotetext{
28 Due to the location of the observatory at the South Pole, scans in the azimuthal direction are equivalent to scans in right ascension; there is no relative rotation between celestial and telescope co-ordinates on the sky.
}

The operator $\star$ is defined via $\mathbf{A} \star \mathbf{B}=A_{y} B_{x}-A_{x} B_{y}$.

This quantity is analogous to estimating " $B$-modes" in cosmic shear experiments. However, many foregrounds (e.g., point sources) have negligible contribution to the curl, and as discussed in the Appendix, gravitational lensing actually generates a nonzero curl power spectrum when using a quadratic estimator. The curl estimate is most useful as a test of our understanding of the fluctuation power in the maps.

\subsubsection{Apodization}

Previous work has dealt with correlations due to sky cuts by setting the noise to be large in cut pixels, and then taking the full pixel-pixel covariance matrix into account (Smith et al. 2007). In a simpler, sub-optimal approach we formulate the estimate initially neglecting the apodization, and then characterize the apodization response using Monte Carlo simulations.

Given that the observed CMB has been convolved with the Fourier transform of an apodization window, $R(\hat{\mathbf{n}})$, i.e.,

$$
T_{R}(\mathbf{l})=\int \frac{d^{2} \mathbf{l}^{\prime}}{(2 \pi)^{2}} T\left(\mathbf{l}^{\prime}\right) R\left(\mathbf{l}-\mathbf{l}^{\prime}\right),
$$

it will possess off-diagonal correlations given by

$$
\left\langle T_{R}\left(\mathbf{l}_{1}\right) T_{R}\left(\mathbf{l}_{2}\right)\right\rangle=\int \frac{d^{2} \mathbf{l}^{\prime}}{(2 \pi)^{2}} C_{\mathbf{l}^{\prime}}^{t} R\left(\mathbf{l}_{1}-\mathbf{l}^{\prime}\right) R\left(\mathbf{l}_{2}+\mathbf{l}^{\prime}\right) .
$$

Running such a CMB field through the lensing estimator will result in a spurious signal at $\mathbf{L} \neq 0$ given by

$$
\begin{aligned}
\langle\hat{d}(\mathbf{L})\rangle= & \frac{A_{\mathbf{L}}}{L} \int \frac{d^{2} \mathbf{l}_{1}}{(2 \pi)^{2}} \int \frac{d^{2} \mathbf{l}^{\prime}}{(2 \pi)^{2}} F\left(\mathbf{l}_{1}, \mathbf{L}-\mathbf{l}_{1}\right) \\
& \times C_{\mathbf{I}^{\prime}}^{t} R\left(\mathbf{l}_{1}-\mathbf{l}^{\prime}\right) R\left(\mathbf{L}-\mathbf{l}_{1}+\mathbf{l}^{\prime}\right) .
\end{aligned}
$$

This signal is a weighted average over the total power in the map, $C_{1}^{t}$, and is present even for an unlensed CMB. In the limit of a very broad window in real space, $R(\mathbf{l}) \rightarrow \delta(\mathbf{l})$ and this signal goes to zero for $\mathbf{L} \neq 0$. For a typical apodization window whose size is tens of degrees, we find that the signal falls to zero quickly with $L$.

In practice, we characterize and remove the mean apodization feature using Monte Carlo simulations. Figure 1 shows the 
deflection map in real space, $d(\hat{\mathbf{n}})$, for one of the SPT fields (RA5H30DEC-55) before and after subtraction of this feature. The feature takes on numerical values $\sim 5$ times a typical $d$ fluctuation. However, as it is a slowly varying function, it is largely decoupled from the multipoles $L>100$ at which we report our lensing results.

\subsection{SPT Lensing Simulations}

To characterize the impact of filtering choices on the lensing signal, we use end-to-end simulations of the data and lensing estimation.

We first generate 100 simulated full-sky lensed CMB realizations using the LensPix package (Lewis 2005), up to a maximum multipole of $l_{\max }=5000$ and at 0.8 resolution. The lensing field is taken to be Gaussian; we address the impact of nonGaussianities in the lensing field, due to the effects of nonlinear growth of density fluctuations, in Section 5.3. We also generate the same number of unlensed full-sky simulations using the HEALPix tools (Górski et al. 2005). ${ }^{29}$ The unlensed CMB simulations are constructed to have the same power spectrum as the lensed simulations, but do not contain the lensing-induced mode couplings. The cosmological parameters are given by the best-fit model for WMAP7 together with the high-multipole measurements of the ACBAR (Reichardt et al. 2009) and QUaD (Brown et al. 2009; Friedman et al. 2009) experiments as found on the LAMBDA Web site. ${ }^{30}$ This model consists of physical baryon density $\Omega_{b} h^{2}=0.02235$, physical cold dark matter density $\Omega_{c} h^{2}=0.1086$, Hubble parameter $H_{0}=70.92 \mathrm{~km} \mathrm{~s}^{-1} \mathrm{Mpc}^{-1}$, optical depth to recombination $\tau=0.0878$, amplitude of primordial scalar fluctuations $A_{s}=2.453 \times 10^{-9}$, and spectral index of primordial scalar fluctuations $n_{s}=0.960$. The latter two quantities are quoted at a reference wavenumber of $k=0.002 \mathrm{Mpc}^{-1}$.

We then project portions of these CMB simulations for each field onto the flat sky, using the oblique equal-area Lambert projection as was done for the real data. To these CMB fields we then add discrete point sources and Gaussian backgrounds consistent with those expected from the SZ effect from galaxy clusters and cosmic infrared background (CIB) fluctuations (Hall et al. 2010; Dunkley et al. 2011; Reichardt et al. 2011; Planck Collaboration et al. 2011b), as was done in K11. We then pass these simulated fields through simulated observations which take into account the detailed time stream filtering applied to the real data, as described in K11. Noise realizations are generated by differencing real SPT observations in two ways: data taken when the telescope is scanning in different directions in azimuth are assigned opposite signs, and then data from separate observations are also assigned random signs.

We then perform lensing reconstruction on these simulated maps, setting the CMB lensing filters to mimic the procedure applied to the real SPT maps, including point-source removal and apodization.

\subsection{Estimating Lensing Power Spectra from Lensing Maps}

In this subsection, we describe our method of estimating the power spectra of the reconstructed deflection maps, including our approach for treating noise bias.

Given a reconstructed map of the deflection field, $\hat{d}(\hat{\mathbf{n}})$, and the associated analytic function, $A_{\mathbf{L}}$, we construct a straightforward lensing power spectrum estimate by averaging the map's

\footnotetext{
29 http://healpix.jpl.nasa.gov

$30 \mathrm{http}: / /$ lambda.gsfc.nasa.gov/product/map/dr4/parameters.cfm
}

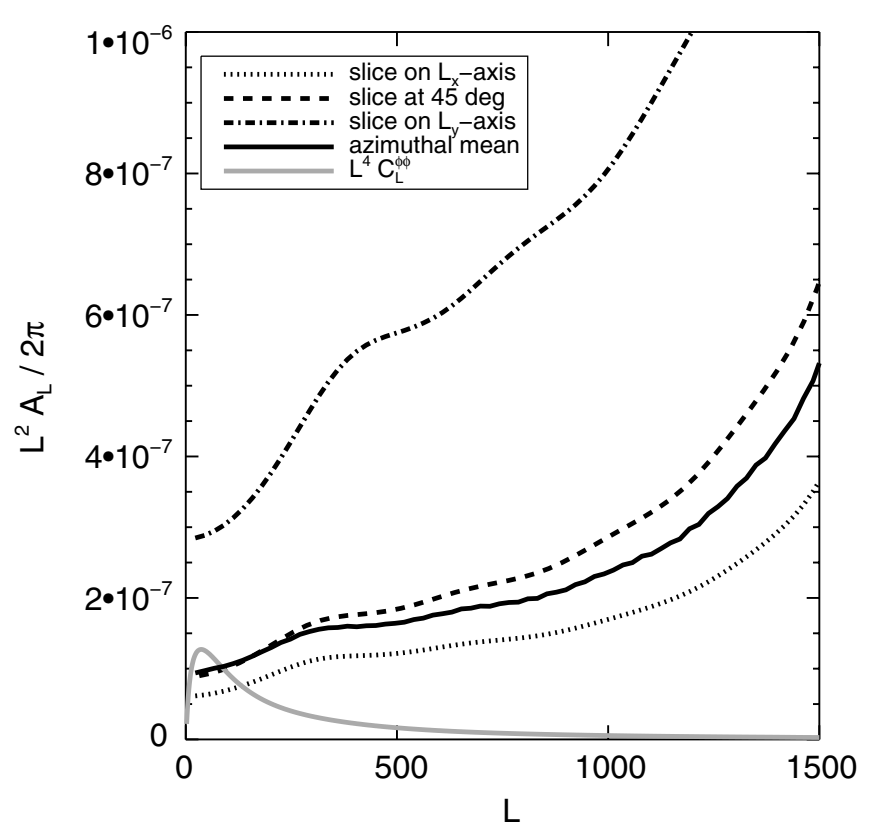

Figure 2. Theoretical lensing reconstruction noise curves $A_{\mathbf{L}}$ (Equation (11)) for SPT map filtering and noise levels, showing slices along the $L_{x}$-direction (dotted), along the $45^{\circ}$ line (dashed) and in the $L_{y}$-direction (dot-dashed). Due to the anisotropic noise statistics and filtering, the lensing data are $\sim 4$ times noisier in the $L_{y}$-direction than in the $L_{x}$-direction. The azimuthally averaged mean theoretical noise curve, given by the thick solid line, is the noise bias which must be subtracted from the lensing power spectrum estimate. Note that the variance of the bandpowers in the lensing power spectrum estimate will not rise as quickly with $L$, as the number of lensing modes to average over is $\propto L$. The fiducial lensing power spectrum $L^{4} C_{L}^{\phi \phi}$ is given by the gray line.

Fourier amplitudes in azimuthal annuli. We down-weight the noisier lensing modes by applying an inverse-variance weight $\propto\left(L^{2} C_{L}^{\phi \phi}+A_{\mathbf{L}}\right)^{-2}$ in the azimuthal average. For $C_{L}^{\phi \phi}$, we use the lensing power spectrum predicted for the fiducial cosmology. The anisotropic theoretical noise level, $A_{\mathbf{L}}$, is shown as slices through the Fourier domain in Figure 2. Because of the filtering of $l_{x}<1000$, as well as the anisotropic SPT noise power, the lensing noise is highly anisotropic in the Fourier domain.

Neglecting covariance between bandpower estimates and assuming that the filters are properly set, the reconstruction is expected to follow (Hu 2001b; Kesden et al. 2003)

$$
\begin{aligned}
\left\langle\hat{d}\left(\mathbf{L}_{1}\right) \hat{d}\left(\mathbf{L}_{2}\right)\right\rangle= & (2 \pi)^{2} \delta\left(\mathbf{L}_{1}+\mathbf{L}_{2}\right)\left(L_{1}^{2} C_{L_{1}}^{\phi \phi}+N_{L}^{(0)}\right) \\
& + \text { (higher-order terms). }
\end{aligned}
$$

The sensitivity to the lensing power spectrum $C_{L}^{\phi \phi}$ originates from the connected part of the CMB four-point function, or trispectrum. The leading noise bias in the reconstruction, $N_{L}^{(0)}$, originates from the unconnected, or purely Gaussian, part of the CMB four-point function. It is present even if lensing reconstruction is performed on an unlensed $\mathrm{CMB}$ field $(\mathrm{Hu}$ 2001b; Amblard et al. 2004). Its theoretical expectation for a field without windowing is shown as the solid black line in Figure 2.

For our analysis, we take two distinct approaches in dealing with this bias.

1. We use full SPT maps of each field to construct lensing maps, and then estimate the lensing power spectrum of each map. This method has the most statistical power, but has a noise bias that must be subtracted. 
2. We split the SPT data into "low-l" and "high- $l$ " bands by applying low-pass and high-pass spatial filters. We estimate two lensing deflection fields, $\hat{d}_{\text {low }}(\mathbf{L})$ and $\hat{d}_{\text {high }}(\mathbf{L})$, from the two bands. We then compute the cross-correlation between these two lensing maps. A lensing detection obtained with this approach will have lower signal to noise, but no Gaussian noise bias.

\subsubsection{All-l Technique}

In this approach, we calculate the expected noise bias for each field using the unlensed end-to-end simulations of Section 4.2. The SPT temperature calibration could potentially vary between the four fields used in this analysis. We calibrate each field by comparing the average temperature power spectrum in the $1200 \leqslant l \leqslant 3000$ range to the temperature power spectrum used in our simulations, which allows us to remove the Gaussian bias with high accuracy. Each field is rescaled at the $1 \%$ level in temperature, and we propagate the residual uncertainty in each field's calibration to the lensing power covariance matrix.

\subsection{2. l-split Technique}

An incorrectly calculated noise bias will lead to an anomalous signal in the measured lensing power spectrum. An alternative to directly characterizing and removing this bias is to construct two maps of the same lensing field, using CMB maps with no modes in common (Hu 2001b; Sherwin \& Das 2010). This can be achieved, for example, by filtering to isolate $\mathrm{CMB}$ multipole ranges in two disjoint annular regions. The estimated cross spectrum between these two reconstructed lensing maps will then contain no Gaussian bias, since the maps have no modes of Gaussian CMB, instrumental noise, or foregrounds in common.

However, due to the smaller number of mode pairs used to construct each of the lensing maps, the resulting lensing power spectrum estimate will have a significantly lower signal-to-noise ratio. We use a Fisher matrix approach to forecast the signal to noise as a function of the split multipole $l_{\text {split }}$. For the SPT noise and filtering, we find that the highest possible detection significance with this split is smaller than that in the all- $l$ analysis by a factor of $\sim 0.38$, for the SPT noise and filtering. We find that this quantity is maximized when the split multipole is set to $l_{\text {split }} \simeq 2300$. This corresponds to a cut slightly higher than the center of the main signal band, $1200<l<3000$.

For each field, we compute lensing maps using only CMB modes with spatial frequencies either of $1200 \leqslant l \leqslant 2200$ or $2300 \leqslant l \leqslant 4000$, with the gap of width $\delta l=100$ between the two annuli being necessary due to the convolution by the finite apodization window. We estimate the lensing signal for each of these maps, and construct a cross power spectrum. We refer to this as the " $l$-split" technique for the remainder of the paper.

\subsubsection{Higher-order Biases}

There are known additional terms in Equation (17) that affect the reconstructed lensing power spectrum. At high $L$, there is a positive bias that arises from correlations in the CMB trispectrum generated by lensing (Kesden et al. 2003). This bias is proportional to the lensing signal, $C_{L}^{\phi \phi}$, though evaluated at a different set of multipoles. It is denoted as $N_{L}^{(1)}$ due to its linear dependence on the lensing power spectrum. This effect also leads to an excess in the power spectrum of the estimated curl field as we show in the Appendix.
There is an additional negative bias which arises at low $L$, due to effects in the reconstruction of the order of $\left(C_{L}^{\phi \phi}\right)^{2}$ (Hu et al. 2007; Hanson et al. 2011). This bias is denoted as $N_{L}^{(2)}$ due to its second-order dependence on the lensing power. This effect is neglected in the formulation of the quadratic estimator, which only considers the lensing operation in the map to linear order in $\phi$ (Equation (5)).

In Section 6.1, we present our detection of lensing in terms of the excess signal compared to $N_{L}^{(0)}$, which is determined from the unlensed simulations. To calibrate the level of lensing power detected, we compare the excess power in the SPT measurements to the excess found in the same analysis of the lensed simulations. Since the lensed and unlensed simulations contain equivalent amounts of Gaussian, on-diagonal CMB power (with $\mathbf{l}_{1}+\mathbf{l}_{2}=0$ ), we label the excess signal seen in the lensed case as the signature of lensing. This simulation-based approach leads to a detection of the effects of lensing which naturally takes the higher-order biases into account, obviating the need to model these biases precisely.

\section{SYSTEMATIC UNCERTAINTIES}

Uncertainties in the underlying cosmology and contributions from foregrounds have been shown to potentially bias the reconstructed lensing power spectrum (Cooray \& Kesden 2003; Amblard et al. 2004; Perotto et al. 2010). In particular, Amblard et al. (2004) have shown that the thermal and kinetic SZ effects can significantly bias the estimates. Here, we re-evaluate these biases, particularly given more recent measurements of the amplitude of the thermal SZ power spectrum, Poisson pointsource power, and clustered point-source power at $150 \mathrm{GHz}$ (Hall et al. 2010; Dunkley et al. 2011; Shirokoff et al. 2011; Reichardt et al. 2011). We also study the impact of nonlinear structures in the universe and beam uncertainties in the context of our analysis approach.

We will show in the results section (Section 6) that the lensing estimator with the highest signal-to-noise ratio, namely, the all- $l$ method, yields a statistical error on the total lensing amplitude of $15 \%$ when applied to our data. Biases on the reconstructed lensing power spectrum which are substantially smaller than this quantity can be safely neglected in our analysis. We show below that none of the possible sources of bias that we consider in this section show evidence for being significant.

\subsection{Foregrounds}

Emission from galaxies and Galactic dust are a possible source of non-Gaussianity, and could in principle be a problem for $\mathrm{CMB}$ lensing reconstructions. In particular, we investigate the impact of infrared and radio galaxies, SZ effects, and Galactic cirrus. We will show that these foregrounds are unlikely to be a substantial source of bias.

\subsubsection{Infrared and Radio Galaxies}

Point sources will affect the estimator in two ways: they will add Gaussian power to the CMB map, and the brightest sources will generate a trispectrum which will lead to an apparent lensing signal. The purely Gaussian component is similar to the experimental noise, and its presence will slightly raise the effective Gaussian noise bias. This is naturally taken into account by our handling of the noise bias described in Section 4.3.1 in the case of the all- $l$ analysis, and is not present as a bias in the $l_{\text {split }}$ analysis. 
The point-source trispectrum is a potential contaminant. Due to the non-locality of the lensing estimator, a bright point source will lead to a spurious signal on all scales. Here, we determine at what flux level the map must be cleaned of bright sources to put the Poisson point-source background into the Gaussian limit for the lensing estimation.

We simulate fields of Poisson-distributed point sources using number count models for dusty, star-forming galaxies given by Negrello et al. (2007), and for radio sources from de Zotti et al. (2005). In the case of the dusty star-forming galaxies, we scale the counts to $150 \mathrm{GHz}$ using the same assumptions on the spectral indices as those described in Hall et al. (2010). These counts agree, up to $100 \mathrm{mJy}$, with the recent measurements of these populations at $150 \mathrm{GHz}$ (Vieira et al. 2010; Marriage et al. 2011; Planck Collaboration et al. 2011a). We generate randomly placed sources with flux values between 0.01 and $10^{3} \mathrm{mJy}$.

We pass these point-source simulations, together with simulated lensed CMB fields and noise realizations, through the lensing estimator. Since we are specifically seeking to isolate the non-Gaussian contribution of the Poisson point sources, for comparison we also pass purely Gaussian fields through the estimator. The Gaussian fields are constructed using the same power spectrum as the non-Gaussian foreground fields, including the effects of source masking. For simplicity, in this section, we use a version of the estimator which is formulated for maps with periodic boundary conditions, bypassing the extra apodization step.

We find that with a flux cut of $10 \mathrm{mJy}$, the uncorrelated trispectrum contribution from Poisson sources is equal to that from equivalent Gaussian power, for both the infrared and radio sources, to within $1 \%$ in the reconstructed lensing power spectrum. Turning the flux cut up to $20 \mathrm{mJy}$, a $~ 5 \%$ bias on the reconstructed lensing power becomes apparent in both the all- $l$ and $l$-split reconstructions. Given that we remove sources in the $150 \mathrm{GHz}$ SPT maps at thresholds of $\sim 10 \mathrm{mJy}$, we conclude that the non-Gaussian contribution of Poisson point sources are an insignificant source of bias on the lensing reconstruction.

We also perform equivalent estimates for the curl signal in these fields. These estimates show negligible signal, indicating that the curl estimate is not a useful check for Poisson foregrounds.

The angular fluctuations in the CIB, as well as the SZ effects, are expected to be correlated with the mass fluctuations responsible for CMB lensing (Song et al. 2003; Cooray \& $\mathrm{Hu}$ 2000). This is because these sources are tracers of the same underlying three-dimensional matter field, and are thought to have a similar distribution in redshift as the CMB lensing redshift kernel (Equation (1)). Clustering of the CIB sources has been detected at SPT wavelengths at high significance (Hall et al. 2010; Hajian et al. 2012; Planck Collaboration et al. 2011b; Shirokoff et al. 2011; Reichardt et al. 2011). The CIB- $\phi$ correlation can potentially bias the lensing estimate (Cooray \& Kesden 2003). To test this possibility, we use two separate lines of investigation: Gaussian random fields that have a CIB field completely correlated with the lensing convergence map, and the simulations of Sehgal et al. (2010).

As a first test for a correlated signal, we assume that both the lensing convergence and the CIB trace the linear density fluctuations in the universe and that both are Gaussian random fields. We assume that the CIB field is completely correlated with the convergence field and normalize the amplitude to match the observations of Reichardt et al. (2011). In this case, we find no measurable bias on the lensing reconstruction.
We also perform an analysis on maps from the IR simulations by Sehgal et al. (2010). Since the simulations were performed, much has been learned about the millimeter-wave properties of the CIB; for example, these simulations assumed a frequency scaling from $353 \mathrm{GHz}$ down to $150 \mathrm{GHz}$ that was more shallow than has been observed (Reichardt et al. 2011; Addison et al. 2012). Using a more appropriate frequency scaling of the dust emissivity than that assumed for the Sehgal et al. (2010) simulations leads to the CIB maps being reduced in amplitude by a factor of 1.7. Scaling the maps by this factor leads to a power spectrum from Poisson-distributed dusty sources that is in excellent agreement with Reichardt et al. (2011).

To study the nature of biases from the Sehgal et al. CIB simulations, we rotate the CIB fields by $90^{\circ}$ to break the correlations between the CIB and lensing fields. We find a small bias ( $<1 \%$ at $L=500$ ) from the CIB sources in the absence of these correlations. Restoring these correlations, the bias in the lensing power spectrum at $L<500$ is found to be $\sim-3 \%-4 \%$, which is smaller than the statistical uncertainty in our analysis (detailed in Section 6).

A complete understanding of the impact of correlations between CIB fluctuations and lensing convergence remains to be determined. The contrast between the results from purely Gaussian simulations (showing no contamination) and the Sehgal simulations $(\sim-3 \%-4 \%$ at low $L)$ demonstrate that careful CIB modeling will be required for future analyses. The Sehgal et al. simulations, while useful for these purposes, have features which make them difficult to interpret. For example, the source counts are lower than observations at $150 \mathrm{GHz}$ (Vieira et al. 2010) between 5 and $10 \mathrm{mJy}$, which is close to the flux cut that we employ; the amplitude of the CIB power spectrum from clustered sources is also lower than that seen in recent measurements; and the finite simulation volume $\left(1 \mathrm{Gpc} h^{-1}\right)$ subtends only $25^{\circ}$ at $z=1$. Larger simulations, created with input from recent observations at millimeter wavelengths, should help gain a better understanding of this systematic effect.

\subsubsection{Thermal and Kinetic Sunyaev-Zel'dovich Effects}

As with the radio and infrared sources, the temperature decrement associated with an unmasked massive SZ cluster leads to a large feature in the reconstructed deflection map. This feature can potentially generate a bias on the reconstructed lensing power spectrum, and correlations of the SZ field with the lensing field could also lead to a bias in the observed lensing power (Cooray \& Kesden 2003).

Our simulated SPT observations, described in Section 4.2, contain Gaussian fluctuations with an SZ power spectrum template. However, they do not contain discrete SZ clusters. Unlike the case for the radio and infrared galaxies, the masking of SZ clusters in the SPT maps does not have an equivalent procedure in our simulated observations. Masking of objects in the data but not in the simulations leads to a small difference in the amount of temperature power in the maps.

Given the possible bias from the thermal SZ signal, we conduct three analyses to assess its importance using two independent thermal SZ simulations, along with an empirical measurement of the importance of SZ masking in the data analysis.

We use the maps of Sehgal et al. (2010), rescaling the amplitude of these maps to match the lower SZ power spectrum seen in measurements (Lueker et al. 2010; Fowler et al. 2010; Reichardt et al. 2011) which were made after these simulations 
were created. As with the radio and infrared sources, we pass these simulations through the lensing estimator, together with simulated CMB and noise fluctuations.

In each case, we compare with a Gaussian field with equivalent power spectrum. We identify two sources of bias from the thermal SZ effect in the Sehgal et al. simulations. The very bright objects contribute a large enough signal in the lensing reconstructions to add a positive bias of $\sim 20 \%$ to the lensing power spectrum without any masking, while correlations of massive galaxy clusters with the large-scale structure responsible for lensing lead to a negative bias of $\sim 10 \%$. Masking of the extremely bright SZ clusters, as is done in the data, reduces the positive bias from the most massive clusters to be less than $10 \%$ of the lensing power spectrum, and the negative bias from correlations with large-scale structure reduces the total bias from the thermal SZ effect to be negligible for this analysis $(<3 \%)$.

The second simulation that we use as an independent test is a $500 \mathrm{Mpc} h^{-1} N$-body+SPH simulation with $1024^{3}$ dark matter as well as gas particles that is performed with a different assumed cosmology (Gottlöber \& Yepes 2007). Snapshots are approximately sampled at each light-crossing time. We then produce maps by randomly rotating and translating each simulation volume and creating an SZ map, then ray tracing over all data cubes adding in each case the SZ effects at the deflected position. After rescaling the maps to agree with the observed SZ power spectrum, we pass these maps through the lensing estimator. In this case, a large bias $(50 \%$ of the lensing power spectrum at $L=700$ ) is generated by the most massive clusters in the map, but masking of the very brightest SZ sources again reduces the bias in the lensing power spectrum to negligible $(\sim 5 \%)$ levels.

The differing results for the two simulations arise from the distinct SZ statistics: the Gottlöber \& Yepes (2007) simulations have an excess of very bright, rare clusters in comparison with those from Sehgal et al. (2010). The main SZ bias in CMB lensing estimation originates from these brightest clusters, due to the scaling of the signal with the fourth power of the temperature.

Given theoretical uncertainties associated with simulations of the thermal SZ (Dunkley et al. 2011; Reichardt et al. 2011), we also take an empirical approach by running the full lensing reconstruction pipeline on SPT maps with differing masking levels. We find that without any masking of clusters, the best-fit lensing amplitude increases by $0.12 \sigma$ ( $2 \%$ of the lensing power spectrum amplitude) compared to the result, presented below, which contains 0.01 clusters masked per square degree. At the more aggressive masking level of 0.07 clusters masked per square degree, the best-fit lensing amplitude decreases by an equivalent amount, $0.12 \sigma$. We therefore conclude that thermal SZ is not a substantial source of bias in this analysis.

We also run simulated kinetic SZ fields through the lensing estimator. Again, we repeat this analysis for the Sehgal et al. as well as smoothed particle hydrodynamics (SPH) simulations. The power spectrum of these fields is consistent with current upper limits (Reichardt et al. 2011). This leads to a bias that is equivalent to a fully Gaussian field with the same power spectrum, to within $1 \%$.

\subsubsection{IR Cirrus}

Diffuse Galactic dust emission is known to be an important foreground for CMB studies. The SPT fields are chosen to minimize Galactic emission, but cirrus emission is detected at $\sim 3 \sigma$ through cross-correlation with maps from the Infrared Astronomical Satellite (Finkbeiner et al. 1999), as described in K11. To test the importance of this cirrus contamination, we subtract a template based on Finkbeiner et al. (1999) from the field that shows the strongest cirrus detection (the RA5H30DEC-55 field), and re-calculate the lensing power spectrum. In no $L$-bin is the result changed by more than $2 \%$, and there is no evidence for a systematic bias. The non-Gaussianity of the Galactic cirrus in our fields is not a serious contaminant for CMB lensing studies at $150 \mathrm{GHz}$.

\subsection{Uncertainty in the Unlensed CMB Temperature Field}

Even if the Gaussian noise bias is perfectly removed, the lensing map is subject to a calibration uncertainty which arises from uncertainty in the CMB power spectrum, as mentioned in $\mathrm{Hu}$ (2001b). This is because the lensing estimate is based on the mode coupling of Equation (5); an uncertainty in the unlensed CMB power spectrum will lead to a multiplicative offset on the reconstructed lensing map.

In this analysis, we enforce a constraint that the power spectrum of the data match that of the simulations, as described in Section 4.3.1. For a fixed theoretical lensed CMB power spectrum, the uncertainty in the theoretical unlensed CMB power spectrum is small. Sample variance between lensed and unlensed power spectra for a given realization is strongly correlated, but in any case would be less than $1 \%$ in amplitude for the sky coverage and $l$-range considered. Uncertainty in the $\mathrm{CMB}$ power spectrum is therefore not a limitation of this analysis.

The effects of primordial non-Gaussianities upon the estimator have been shown to be negligible, being two orders of magnitude smaller than the first-order bias of Kesden et al. (2003) for non-Gaussianity parameters $f_{\mathrm{NL}}$ and $g_{\mathrm{NL}}$ consistent with current upper limits (Lesgourgues et al. 2005).

\subsection{Effects from Nonlinear Growth of Structure}

Interaction between the angular scales of the gradient and lens (Equation (10)) can lead to a negative bias of the lensing reconstruction (Hu et al. 2007; Hanson et al. 2011). This effect is expected to increase in the presence of nonlinear structures like clusters and filaments. To determine whether our simulation pipeline using Gaussian random fields of matter fluctuations (described in Section 4.2) leads to an unbiased reconstruction, we run a cosmological $N$-body simulation of gravitationally interacting dark matter particles, using the Gadget- 2 code. ${ }^{31}$ The simulated cube is $1000 \mathrm{Mpc} h^{-1}$ on each side with $1024^{3}$ particles. We again produce ray-tracing simulations of CMB lensing in maps $15^{\circ} \times 15^{\circ}$ on a side that start from a regular grid near the observer and deflect each ray at the interpolated positions at each projected plane. The resulting lensing potential has excess small-scale power as expected in nonlinear structure formation, and the lensed temperature power spectrum is found to be consistent with the nonlinear lensing option of CAMB (Lewis et al. 2000) out to $l \simeq 5000$. This confirms convergence of the resolution of our dark matter simulation on all scales relevant for the SPT lensing reconstruction. The lensing estimator (Section 2) is then applied to the lensed maps and the reconstructed lensing power spectrum compared to that of the input. The procedure is repeated for 100 maps produced from different randomly chosen translations and rotations of the simulation volumes along

\footnotetext{
31 http://www.mpa-garching.mpg.de/gadget/right.html
} 


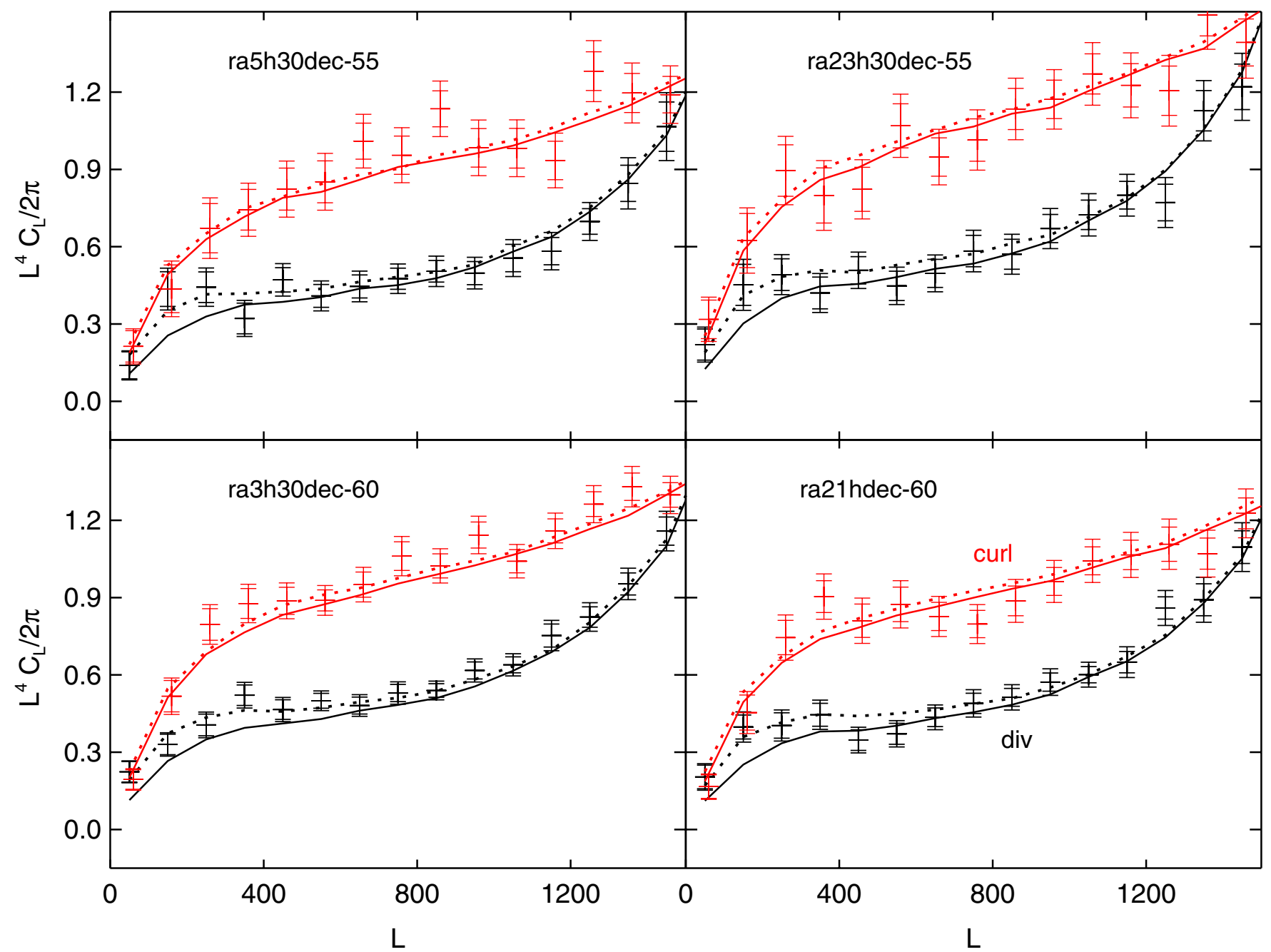

Figure 3. Individual all- $l$ raw power spectra for each field for the main lensing signal (the divergence; bottom: black points in each panel) and the curl component (top: red points in each panel). Curves show the results of the lensed and unlensed simulations; i.e., the lower curves show the Gaussian noise biases estimated from simulations and the upper curves show the sum of the noise bias and the expected lensing signal in our fiducial cosmological model. The extra ticks on the error bars show the impact of the correlated covariance arising from the uncertainty in the Gaussian noise bias subtraction.

(A color version of this figure is available in the online journal.)

the line of sight. We find a deviation of the second-order lensing bias $N_{L}^{(2)}$ from the Gaussian case with magnitude equal to a few percent of the total reconstructed power. This bias is insignificant compared to sample variance for the sizes of the SPT fields.

\subsection{Beam Uncertainties}

Here, we address the uncertainty in our analysis due to the uncertainty in the SPT beam profiles. The simulated observations convolve the sky by the SPT beams. If the beam used in this convolution differs from the true SPT beam, then the beamconvolved sky power, and thus $N_{L}^{(0)}$ bias, will differ between the data and the simulations. This would result in a bias in our $N_{L}^{(0)}$-subtracted $C_{L}^{\phi \phi}$.

To first order, this effect is removed when we recalibrate the data maps such that their average beam-convolved temperature power spectra are equal to the simulated beam-convolved power spectra. However, there is a residual uncertainty due to the tilt of the beam uncertainty across the $1200<l<3000$ range. We have checked that the effect of this tilt is small. If we repeat the analysis using simulated beams that differ from the nominal beams by a $1 \sigma$ beam uncertainty, then we find that the bestfit lensing amplitude shifts only by $-0.4 \%$, or $-0.03 \sigma$. We conclude that the uncertainty in the beam has a negligible effect on this analysis.

\section{RESULTS}

Two types of results are reported below. First, the amplitude of the lensing signal is compared with expectations from our simulations, which are performed at a single point in cosmological parameter space. We then explore the cosmological parameter space allowed by current cosmological probes, using the lensing data to both better constrain cosmological parameters and characterize the amplitude of gravitational lensing compared to expectations from the ensemble of allowed cosmological models.

\subsection{Measuring the Lensing Amplitude at a Reference $\Lambda$ CDM Cosmology}

The raw, unnormalized power spectra of the estimated deflection maps are shown in Figure 3. The spectra are dominated by the lowest-order noise bias, and, additionally, have not been 

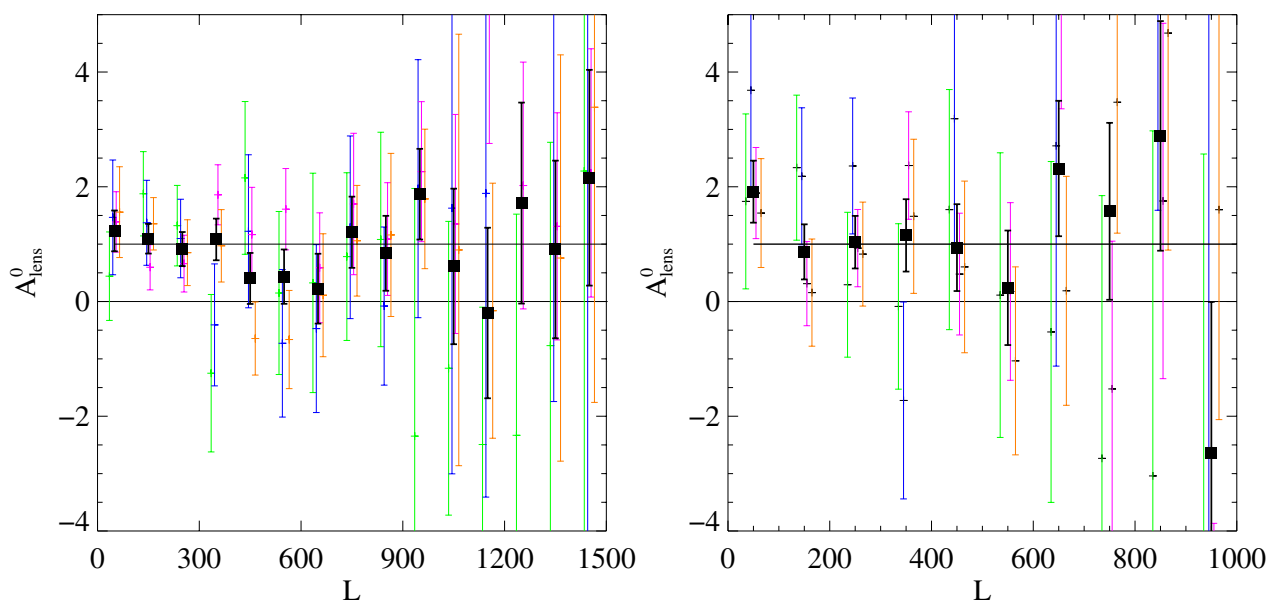

Figure 4. Ratio of the power excess measured in the SPT data compared to the power excess from lensed simulations. The left panel uses all- $l$ maps, while the right panel uses the $l$-split method of using disjoint annuli in $l$ space to avoid a noise bias. The horizontal lines indicate lensing amplitudes of zero and one. Each field is shown as a different color, offset in $L$ for clarity: the RA5H30DEC-55 field in green, the RA23H30DEC-55 field in blue, the RA3H30DEC-60 field in magenta, and the RA21 HDEC-60 field in orange. The heavy black points show the combined best-fit estimate of the lensing amplitude. Note the expanded scale in the right panel; the $l$-split method has less statistical power. No lensing is excluded at $6.3 \sigma$ (left) and 3.9 $\sigma$ (right).

(A color version of this figure is available in the online journal.)

corrected for the effects of the windowing or the higher-order biases. The noise bias is substantial, but it is also clear that the SPT data show an excess in all fields over the unlensed prediction. The curl estimator also shows a preference for lensing, and demonstrates that the Gaussian noise in the SPT maps is well understood.

After subtracting the expected noise bias, we compare the measured excess in the divergence estimator to that seen in the lensed simulations in the left panel of Figure 4. The relative bandpowers are shown as an $L$-dependent scale factor

$$
A_{\text {lens }}^{0}(L)=\frac{\hat{C}_{L}^{\text {data }}-\hat{N}_{L}^{(0)}}{\hat{C}_{L}^{\text {sim }}-\hat{N}_{L}^{(0)}} .
$$

Here, $\hat{C}_{L}^{\text {data }}$ is the raw power spectrum of the reconstructed lensing deflection field; $\hat{C}_{L}^{\text {sim }}$ is the field-dependent raw power spectrum of the lensed simulations; and $\hat{N}_{L}^{(0)}$ is the field-dependent noise bias, which is obtained by performing equivalent reconstructions on the unlensed simulations. The superscript $(0)$ refers to this being the amplitude of the lensing signal relative to the template provided by our simulations. With this definition, $A_{\text {lens }}^{0}(L)=1$ corresponds to the amplitude of the lensing signal in the simulations; $A_{\text {lens }}^{0}(L)=0$ corresponds to no lensing signal. We show this quantity for the all- $l$ analysis in the left panel of Figure 4; evidence for lensing can clearly be seen. The right panel of Figure 4 shows the same quantity for the $l$-split technique, which has no Gaussian noise bias needing removal.

We then fit the measured $A_{\text {lens }}^{0}(L)$ to the model of an $L$-independent lensing amplitude $A_{\text {lens }}^{0}$ which scales the amplitude of the lensing power spectrum in our fiducial cosmology. We assume a Gaussian likelihood function of the form

$$
\begin{aligned}
-2 \ln \mathcal{L}\left(A_{\text {lens }}^{0}\right)= & \ln \operatorname{det}(\mathbf{C})+\sum_{L L^{\prime}}\left(A_{\text {lens }}^{0}(L)-A_{\text {lens }}^{0}\right) \\
& \times \mathbf{C}_{L L^{\prime}}^{-1}\left(A_{\text {lens }}^{0}\left(L^{\prime}\right)-A_{\text {lens }}^{0}\right) .
\end{aligned}
$$

We obtain an approximation to the bandpower covariance matrix $\mathbf{C}$ using 2000 lensed flat-sky simulations which include apodization for the RA5H30DEC-55field. This large number of

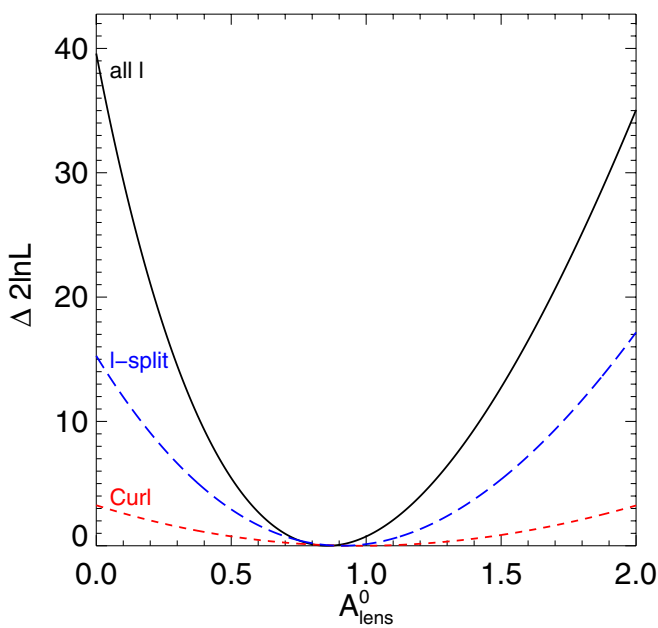

Figure 5. Change in $2 \ln L$ compared to best fit for the SPT lensing power spectrum, when the fiducial lensing power spectrum is multiplied by a lensing scale factor $A_{\text {lens }}^{0}$. A strong detection is evidenced for both the less-sensitive $l$-split method (blue, long dashed line) and the more-sensitive all- $l$ technique (black, solid line). The curl signal in the data (red, short dashed) shows mild evidence for lensing.

(A color version of this figure is available in the online journal.)

simulations is necessary due to the large scatter in the offdiagonal terms. The bands are correlated at the $15 \%-20 \%$ level; the shape of the off-diagonal elements in the covariance matrix is found to be similar to that obtained by Kesden et al. (2003) and Hanson et al. (2011), using mode-counting arguments. We additionally account for sample variance in the lensing amplitude by scaling the diagonals of the covariance matrix by a factor $\propto\left[\left(A_{\text {lens }}^{0} C_{L}^{\phi \phi}+N_{L}^{(0)}\right) /\left(C_{L}^{\phi \phi}+N_{L}^{(0)}\right)\right]^{2}$.

To test the assumption of Gaussianity in the likelihood function, we also compare with an offset-lognormal likelihood function (Bond et al. 2000). We find equivalence with the two approaches in both the best-fit point and the width of the likelihood curves.

Figure 5 shows the total likelihood for the fields as a function of $A_{\text {lens }}^{0}$ and indicates a robust detection of lensing power. No lensing is excluded at $3.9 \sigma$ using the $l$-split approach, and at $6.3 \sigma$ using the all- $l$ approach. These quantities are quoted in 
Table 1

$\chi^{2}$ Values and Maximum Likelihood Fits for Each Field

\begin{tabular}{|c|c|c|c|c|c|c|c|c|c|}
\hline \multirow[t]{2}{*}{ Field Name } & \multicolumn{3}{|c|}{$\chi^{2}\left(A_{\text {lens }}^{0}=1\right)$} & \multicolumn{3}{|c|}{$\chi^{2}\left(A_{\text {lens }}^{0}=0\right)$} & \multicolumn{3}{|c|}{ Best-fit $A_{\text {lens }}^{0}$} \\
\hline & Div (all- $l$ ) & Div (l-split) & Curl & Div (all-l) & Div ( $l$-split) & Curl & Div (all- $l$ ) & Div ( $l$-split) & Curl \\
\hline RA 5H30DEC-55 & 12.0 & 4.0 & 18.7 & 31.0 & 5.1 & 20.0 & $1.40 \pm 0.45$ & $0.58 \pm 0.60$ & $1.1_{-1.1}^{+1.3}$ \\
\hline RA23H30DEC-55 & 14.3 & 14.3 & 12.0 & 21.7 & 22.4 & 12.3 & $0.77 \pm 0.39$ & $1.92 \pm 0.69$ & $0.2_{-0.2}^{+1.3}$ \\
\hline RA3H30DEC-60 & 10.6 & 13.9 & 13.4 & 25.2 & 20.6 & 17.9 & $0.84 \pm 0.28$ & $0.90 \pm 0.38$ & $2.0_{-0.9}^{+0.9}$ \\
\hline RA21HDEC-60 & 22.5 & 5.1 & 16.3 & 31.3 & 6.7 & 16.3 & $0.63 \pm 0.26$ & $0.60 \pm 0.47$ & $0.1_{-0.1}^{+1.0}$ \\
\hline Total (number of points) & $59.4(56)$ & $37.2(36)$ & $60.4(56)$ & $109.3(56)$ & $54.8(36)$ & $66.6(56)$ & $0.86 \pm 0.16$ & $0.91 \pm 0.25$ & $0.98 \pm 0.55$ \\
\hline
\end{tabular}

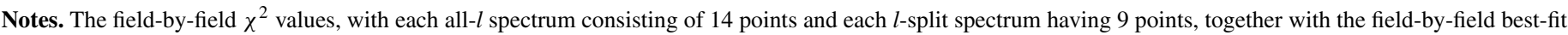
$A_{\text {lens }}^{0}$ for the different spectra. The curl uncertainties are asymmetric within each field because we have assumed $A_{\text {lens }}^{0} \geqslant 0$.

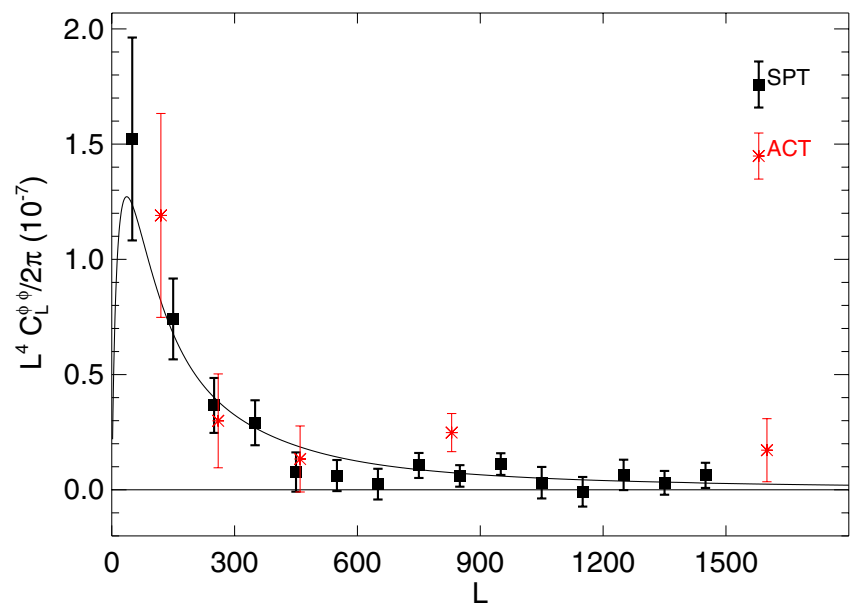

Figure 6. Comparison of the derived lensing bandpowers from SPT and ACT (Das et al. 2011b). Although we show the lowest- $L$ data point, centered at $L=50$, we do not use this point in our fits due to the possible interaction with the subtraction of the apodization feature (Section 4.1.2) on this large scale. The solid curve is not a fit to the data; rather, it is the lensing power spectrum in our fiducial $\Lambda \mathrm{CDM}$ cosmology, corresponding to $A_{\text {lens }}^{0}=1$.

(A color version of this figure is available in the online journal.)

terms of the difference in the likelihood function between zero and the best-fit $A_{\text {lens }}^{0}$, taking the total likelihood as the sum of likelihoods for the individual fields. Using the divergence signal in the all- $l$ maps, the best-fit lensing amplitude is found to be $0.86 \pm 0.16$. A substantial component of this uncertainty comes from the uncertainty associated with the $N_{L}^{(0)}$ removal; in the absence of this uncertainty, the error bar would be \pm 0.11 .

The values of $\chi^{2}$, the second term in Equation (19), are shown in Table 1 for the individual fields. For $A_{\text {lens }}^{0}=1$ (not the best fit), the highest $\chi^{2}$ value for any field still has a $7 \%$ probability of observing a higher value, and the total $\chi^{2}$ for all 56 points has a probability of $35 \%$ of observing a higher value. In contrast, all of the fields have higher $\chi^{2}$ for a model with no gravitational lensing, and the sum of the fields has a $\chi^{2}$ with a probability of observing a higher value of $8 \times 10^{-5}$.

The $l$-split technique shows a clear lensing detection with $A_{\text {lens }}^{0}=0.91 \pm 0.25$. As expected, the signal to noise is substantially lower, but recall that this lensing power spectrum does not suffer from the issues of noise bias that present a challenge for the result that uses the all- $l$ maps.

Using only the curl signal in the all- $l$ maps, we find a value for the lensing amplitude which is consistent with those from the other approaches, $A_{\text {lens }}^{0}=0.98 \pm 0.55$. The lensing signal in this mode is due to the equivalent of the $N_{L}^{(1)}$ bias mentioned above and discussed in more detail in the Appendix.
Table 2

SPT Lensing Bandpowers

\begin{tabular}{lcc}
\hline \hline$L$ & $L^{4} C_{L}^{\phi \phi} / 2 \pi / 10^{-7}$ & $\sigma\left(L^{4} C_{L}^{\phi \phi} / 2 \pi / 10^{-7}\right)$ \\
\hline 150 & 0.741 & 0.175 \\
250 & 0.366 & 0.119 \\
350 & 0.291 & 0.098 \\
450 & 0.077 & 0.085 \\
550 & 0.062 & 0.067 \\
650 & 0.025 & 0.067 \\
750 & 0.106 & 0.054 \\
850 & 0.060 & 0.047 \\
950 & 0.112 & 0.047 \\
1050 & 0.031 & 0.068 \\
1150 & -0.009 & 0.064 \\
1250 & 0.065 & 0.066 \\
1350 & 0.030 & 0.052 \\
1450 & 0.063 & 0.055 \\
\hline
\end{tabular}

Notes. The lensing bandpowers, as shown in Figure 6. Each value represents a band of width $\Delta L=100$, centered at the given value of $L$. The final column shows the error within the given band, obtained from simulations. The bandpowers of the lensing convergence $\kappa$ are related to those of the potential $\phi$ according to $C_{L}^{\kappa \kappa}=(1 / 4) L^{4} C_{L}^{\phi \phi}$.

The majority of the uncertainty in the measurement originates from the CMB fluctuations, rather than instrumental noise fluctuations. If the analysis we have performed were repeated on data from an experiment with temperature noise levels that are $10 \%$ higher than the $18 \mu \mathrm{K}^{\prime}, \mathrm{CMB}$ lensing would be detected with a significance that is decreased by $\sim 4 \%$. Substantial gains in signal-to-noise ratio can instead be obtained by observing more area of CMB sky.

Figure 6 shows the product of the derived lensing amplitudes as a function of $L$ and the reference lensing power spectrum used in our simulations, $A_{\text {lens }}^{0}(L) C_{L}^{\phi \phi}$. This represents our best estimate of the lensing power spectrum. These bandpowers are also shown in Table 2. The $N_{L}^{(1)}$ and $N_{L}^{(2)}$ biases lead to non-local distortions of the lensing power spectrum. However, if the shape and amplitude of the true lensing power spectrum is similar to that in the assumed power spectrum, then the true biases will not be significantly different than what is assumed.

\subsection{Cosmological Parameter Estimation with Extra Information from Lensing}

The power spectrum of the CMB lensing potential is sensitive to total matter fluctuations over a wide redshift range (peaking at $z \sim 2$ ), and mainly on scales which are in the linear regime 
$\left(k \sim 0.05 h \mathrm{Mpc}^{-1}\right)$. A measurement of this power spectrum can therefore constrain physics which affects growth on these scales, as well as provide a distance measure to these redshifts (Kaplinghat et al. 2003; Smith et al. 2006; Lesgourgues et al. 2006; de Putter et al. 2009). The first cosmological constraints from the lensing power spectrum were produced with the ACT lensing reconstruction by Sherwin et al. (2011). When combined with data from WMAP, the lensing data showed a preference for spatial flatness, and found a nonzero dark energy density at $3.2 \sigma$. We first discuss the constraining power from the SPT data in the $\Lambda \mathrm{CDM}$ parameter space. We then quantify the improvement in four additional parameters, which we allow to vary: the amplitude of the lensing signal $A_{\text {lens }}$, the spatial curvature of the universe $\Omega_{k}$, a nonzero sum of neutrino masses $\Sigma m_{v}$, and the dark energy equation of state parameter $w$.

One complicating factor in using lensing measurements to obtain precision cosmological constraints is the nontrivial scaling of the higher-order biases with cosmological parameters (Kesden et al. 2003; Amblard et al. 2004; Hanson et al. 2011).

In our approach, we obtain an effective $L$-dependent lensing amplitude $A_{\text {lens }}^{0}(L)$ seen in the data relative to its expectation from simulations (as shown in Figure 4). We then multiply by the fiducial lensing power spectrum $C_{L}^{\phi \phi}$ used in the simulations. If the true cosmological parameters were exactly equal to those assumed in our simulations, then the higher-order biases would be completely accounted for in this approach. To consider different cosmological parameters, we must therefore estimate the different scalings with cosmological parameters for these higher-order effects.

The leading-order bias $N_{L}^{(1)}$ at a given multipole $L$ is given as an integration over the lensing power spectrum at other multipoles. A parameter which scales the amplitude of the lensing signal, such as the scalar spectral amplitude $A_{s}$, will thus affect $C_{L}^{\phi \phi}$ and $N_{L}^{(1)}$ in the same way. However, a parameter which affects the shape of the spectrum in a nontrivial way will affect $C_{L}^{\phi \phi}$ and $N_{L}^{(1)}$ differently (Hanson et al. 2011). We note that the measurements at low $L$, which contain the highest signalto-noise ratio, are dominated by $C_{L}^{\phi \phi}$ (after the subtraction of $\left.N_{L}^{(0)}\right)$. The size of $N_{L}^{(1)}$ becomes $\gtrsim 50 \%$ of the signal at $L=$ 1000; however, the signal-to-noise ratio per band also decreases at high $L$.

To estimate the impact of the scaling of $N_{L}^{(1)}$ with parameters, we analytically compute $N_{L}^{(1)}$ at a grid of points in parameter space. The calculation of the $N_{L}^{(1)}$ bias is a CPU-intensive fourdimensional integral in the Fourier domain for each value of $L$ considered. For simplicity, we therefore compute $N_{L}^{(1)}$ assuming isotropic noise fluctuations. This allows us to evaluate $N_{L}^{(1)}$ on a one-dimensional line $L$, rather than at a two-dimensional grid of points as would be necessary if considering the anisotropic SPT noise. We then numerically evaluate the derivatives

$$
B_{\alpha} \equiv\left|\frac{d}{d p_{\alpha}} \ln \left(\frac{m(L)^{2} L^{4} C_{L}^{\phi \phi}+L^{2} N_{L}^{(1)}}{m(L)^{2} L^{4} C_{L}^{\phi \phi}}\right)\right| \sigma_{p_{\alpha}}
$$

The factor $m(L)^{2}$ encapsulates the calibration offset in the lensing estimate due to uncertainty in the unlensed CMB power spectrum, discussed in Section 5.2; it is equal to unity if the unlensed CMB power spectrum is equal to its assumed value. The set of parameters $p_{\alpha}$ which we vary consists of $\left(\Omega_{b} h^{2}, \Omega_{c} h^{2}, H_{0}, \tau, A_{s}, n_{s}, \Sigma m_{v}, \Omega_{k}, w\right)$. As the final step, we multiply by the cosmologically allowed $1 \sigma$ range in the given parameter, $\sigma_{p_{\alpha}}$. We find that the logarithmic derivative $B_{\alpha}$ is less than 0.02 for $L<1300$ for all parameters considered. The two bins at higher $L$ constitute only $3.2 \%$ of the total SPT lensing $\chi^{2}$, and contain effectively negligible weight in parameter fits. $N_{L}^{(1)}$ can thus be treated as a transfer-function effect on the lensing modes used in the current analysis.

The second-order, negative bias, $N_{L}^{(2)}$, appears on the largest scales. The SPT lensing bandpowers correspond to scales smaller than a full-sky experiment, such as Planck. Using simulations, we find that at $L=150$, the lowest $L$ at which we report our results, its value is approximately $\left|N_{150}^{(2)}\right|=0.60 \sigma_{150}$. Here, $\sigma_{150}$ denotes the uncertainty in the reported band at $L=150$. At $L=250$, its value is $\left|N_{250}^{(2)}\right|=0.17 \sigma_{250}$. In our approach, the majority of the effect of $N_{L}^{(2)}$ is removed by scaling to its value in the reference cosmology. Since the lensing amplitude is measured at the $\sim 20 \%$ level from the other bands, the uncertainty in this rescaling is small (on the order of $\sim 0.2 \times 0.6 \sigma_{150}=0.12 \sigma_{150}$ for the band at $L=150$, and smaller at higher multipoles). We thus neglect the effect of $N_{L}^{(2)}$ in the following analysis.

To explore high-dimensional parameter volumes, we use Monte Carlo Markov chain (MCMC) techniques (Christensen et al. 2001; Lewis \& Bridle 2002). Rather than computing new Markov chains, we importance-sample existing chains using the SPT lensing likelihood (e.g., Appendix B of Lewis \& Bridle 2002). The chains we use were generated for the CMB temperature power spectrum analysis of K11 using a modified version of the CosmoMC package. They provide full explorations of the allowed parameter volumes for various models, constrained by the WMAP7 CMB power spectrum measurements (Komatsu et al. 2011). In some cases, we also consider the impact of including the SPT high-l CMB temperature power spectrum measurements of K11.

The base parameter set varied in the chains consists of $\left(\Omega_{b} h^{2}, \Omega_{c} h^{2}, \theta_{s}, \tau, A_{s}, n_{s}\right)$, where $\theta_{s}$ is the angular scale subtended by the sound horizon at the CMB recombination surface. The parameters describing the power spectrum of primordial fluctuations, $A_{s}$ and $n_{s}$, are defined relative to a reference wavenumber of $k=0.002 \mathrm{Mpc}^{-1}$, as is chosen in the analysis of the WMAP team (Komatsu et al. 2011). In the case of the chains which are computed with K11 data, the amplitudes of the three sources of foreground fluctuations which become important on small angular scales are also varied and marginalized over. These consist of the amplitude of the power spectrum of clustered infrared galaxies; the amplitude of the power spectrum associated with the Poisson, or shot noise, nature of the galaxy distribution; and the amplitude of the power spectrum of SZ fluctuations. All cosmological parameters are assigned flat priors, with the exception of the logarithmic prior assigned to $A_{s}$. Foreground parameters have priors based on the measurements in Shirokoff et al. (2011), as described in K11.

We generate a lensing power spectrum for each point in these chains. The calculation of accurate theoretical lensing power spectra is CPU-intensive. For efficiency, we first calculate lensing power spectra for $\sim 10^{4}$ points in a chain using the CAMB software package (Lewis et al. 2000). For this step, we use the parameter chains provided by the WMAP team as a training set. We then use this information to interpolate power spectra at other points in the parameter space. We perform a principal component analysis on the training set, keeping the first 12 modes. We then perform a linear fit to the amplitudes of 
Table 3

Constraints on Cosmological Parameters using SPT Lensing Bandpowers

\begin{tabular}{|c|c|c|c|c|c|c|c|}
\hline $\begin{array}{l}\text { Model and } \\
\text { Parameter }\end{array}$ & & WMAP7 & $\begin{array}{l}\text { WMAP7 + } \\
\text { SPTlens }\end{array}$ & WMAP7 + K11 & $\begin{array}{l}\text { WMAP7 + K11 + } \\
\text { SPTlens }^{\mathrm{a}}\end{array}$ & $\mathrm{WMAP} 7+H_{0}$ & $\begin{array}{c}\text { WMAP7 }+H_{0}+ \\
\text { SPTlens }\end{array}$ \\
\hline $\begin{array}{l}\Lambda \mathrm{CDM} \\
\text { (six parameters) }\end{array}$ & $\begin{array}{c}\sigma_{8} \\
\Omega_{c} h^{2} \\
\end{array}$ & $\begin{array}{c}0.821 \pm 0.029 \\
0.1125 \pm 0.0054 \\
\end{array}$ & $\begin{array}{c}0.810 \pm 0.026 \\
0.1103 \pm 0.0047\end{array}$ & $\begin{array}{c}0.814 \pm 0.024 \\
0.1117 \pm 0.0048\end{array}$ & $\begin{array}{c}0.806 \pm 0.022 \\
0.1102 \pm 0.0042\end{array}$ & $\begin{array}{c}0.809 \pm 0.027 \\
0.1091 \pm 0.0043\end{array}$ & $\begin{array}{c}0.803 \pm 0.025 \\
0.1081 \pm 0.0039 \\
\end{array}$ \\
\hline $\begin{array}{l}\Lambda \mathrm{CDM}+A_{\text {lens }} \\
\text { (seven parameters) }\end{array}$ & $A_{\text {lens }}$ & $1.13 \pm 0.98$ & $0.90 \pm 0.19$ & $0.92 \pm 0.23$ & $0.90 \pm 0.15$ & $1.26 \pm 1.03$ & $0.95 \pm 0.19$ \\
\hline $\begin{array}{l}\Lambda \mathrm{CDM}+\Omega_{k} \\
\text { (seven parameters) }\end{array}$ & $\begin{array}{l}\Omega_{k} \\
H_{0} \\
\Omega_{\Lambda}\end{array}$ & $\begin{array}{c}-0.0545 \pm 0.0670 \\
57.6 \pm 13.8 \\
0.561 \pm 0.193\end{array}$ & $\begin{array}{c}-0.0014 \pm 0.0172 \\
72.3 \pm 9.3 \\
0.734 \pm 0.056\end{array}$ & $\begin{array}{c}-0.0150 \pm 0.0257 \\
66.4 \pm 9.8 \\
0.689 \pm 0.081\end{array}$ & $\begin{array}{c}-0.0015 \pm 0.0146 \\
72.2 \pm 7.9 \\
0.738 \pm 0.046\end{array}$ & $\begin{array}{c}0.0045 \pm 0.0053 \\
73.3 \pm 2.4 \\
0.744 \pm 0.019\end{array}$ & $\begin{array}{c}0.0042 \pm 0.0052 \\
73.6 \pm 2.4 \\
0.749 \pm 0.017 \\
\end{array}$ \\
\hline $\begin{array}{l}\Lambda \mathrm{CDM}+\Sigma m_{v} \\
\text { (seven parameters) }\end{array}$ & $\begin{array}{c}\Sigma m_{v}(\mathrm{eV}) \\
\sigma_{8} \\
\Omega_{c} h^{2}\end{array}$ & $\begin{array}{c}<1.10(95 \% \text { CL }) \\
0.726 \pm 0.070 \\
0.1187 \pm 0.0072\end{array}$ & $\begin{array}{c}<1.17 \\
0.709 \pm 0.066 \\
0.1184 \pm 0.0073\end{array}$ & $\begin{array}{c}<1.34 \\
0.688 \pm 0.072 \\
0.1208 \pm 0.0074\end{array}$ & $\begin{array}{c}<1.37 \\
0.677 \pm 0.068 \\
0.1212 \pm 0.0075\end{array}$ & $\begin{array}{c}<0.36 \\
0.774 \pm 0.041 \\
0.1094 \pm 0.0043\end{array}$ & $\begin{array}{c}<0.38 \\
0.768 \pm 0.039 \\
0.1088 \pm 0.0039 \\
\end{array}$ \\
\hline $\begin{array}{l}\text { wCDM } \\
\text { (seven parameters) }\end{array}$ & $\begin{array}{c}w \\
\sigma_{8} \\
\Omega_{c} h^{2}\end{array}$ & $\begin{array}{c}-1.120 \pm 0.420 \\
0.854 \pm 0.143 \\
0.1132 \pm 0.0056\end{array}$ & $\begin{aligned}-1.040 & \pm 0.399 \\
0.818 & \pm 0.131 \\
0.1109 & \pm 0.0048\end{aligned}$ & $\begin{aligned}-1.160 & \pm 0.363 \\
0.863 & \pm 0.120 \\
0.1123 & \pm 0.0047\end{aligned}$ & $\begin{aligned}-1.105 & \pm 0.352 \\
0.838 & \pm 0.115 \\
0.1107 & \pm 0.0042\end{aligned}$ & $\begin{array}{c}-1.126 \pm 0.111 \\
0.863 \pm 0.053 \\
0.1135 \pm 0.0056\end{array}$ & $\begin{aligned}-1.087 & \pm 0.096 \\
0.838 & \pm 0.045 \\
0.1108 & \pm 0.0047\end{aligned}$ \\
\hline
\end{tabular}

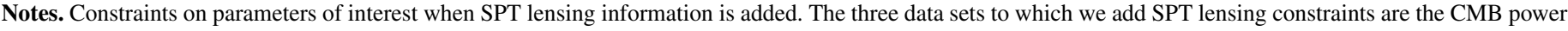

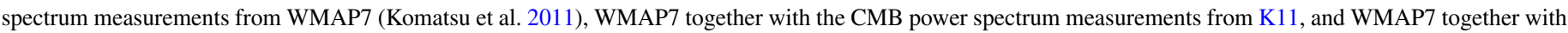

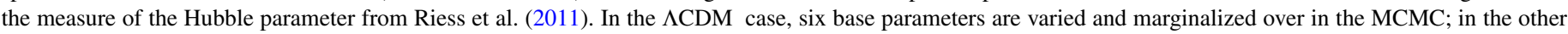

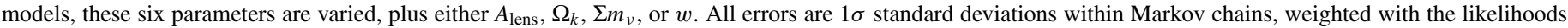
for the given data sets. The Hubble parameter is quoted in units of $\mathrm{km} \mathrm{s}^{-1} \mathrm{Mpc}^{-1}$.

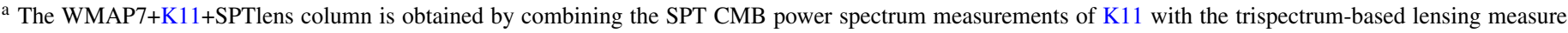
performed in this paper, and is subject to the validity of neglecting the covariance between the two measures (O. Zahn et al. 2012, in preparation).

these modes as a function of cosmological parameters. With this linear fit for the mode amplitudes we can construct a lensing power spectrum for any set of cosmological parameters. We find the fit (at $L<2000$ ) to have an rms difference of less than $1 \%$ from the CAMB-computed power spectrum for cosmological models within the WMAP7-allowed $3 \sigma$ parameter space.

For each of these theoretical lensing power spectra, we then calculate the likelihood of the SPT lensing data. We assume a Gaussian likelihood function, consisting of the SPT bandpowers shown in Figure 6 together with the covariance matrix used to constrain $A_{\text {lens }}$ above. Unlike when fitting for the template amplitude $A_{\text {lens }}^{0}$, in which we fit for each field separately, here we fit directly to the field-combined bandpowers.

In the Metropolis-Hastings technique of MCMC integration (Metropolis et al. 1953), which is used for the chains considered in this paper, each location in parameter space examined by the chain is assigned a weight according to the number of iterations that the chain remained at that point. To include the SPT lensing measurements, we calculate new weights by multiplying this weight by the SPT lensing likelihood. We can then compute statistics, such as marginalized oneand two-dimensional parameter distributions, by replacing the original weights with these new weights. The constraints on cosmological parameters presented in the remainder of this section are quoted as the means and variances of the distributions obtained using these new weights. The constraints we obtain for each model are summarized in Table 3.

\subsection{1. $\Lambda C D M$}

The first cosmological model we consider is the spatially flat, power-law $\Lambda \mathrm{CDM}$ model (with the lensing amplitude set to unity). The parameter constraints which are most improved when the SPT lensing data are added to the WMAP7-allowed $\Lambda C D M$ parameter volume are those of the cold dark matter density and the two parameters related to the primordial scalar

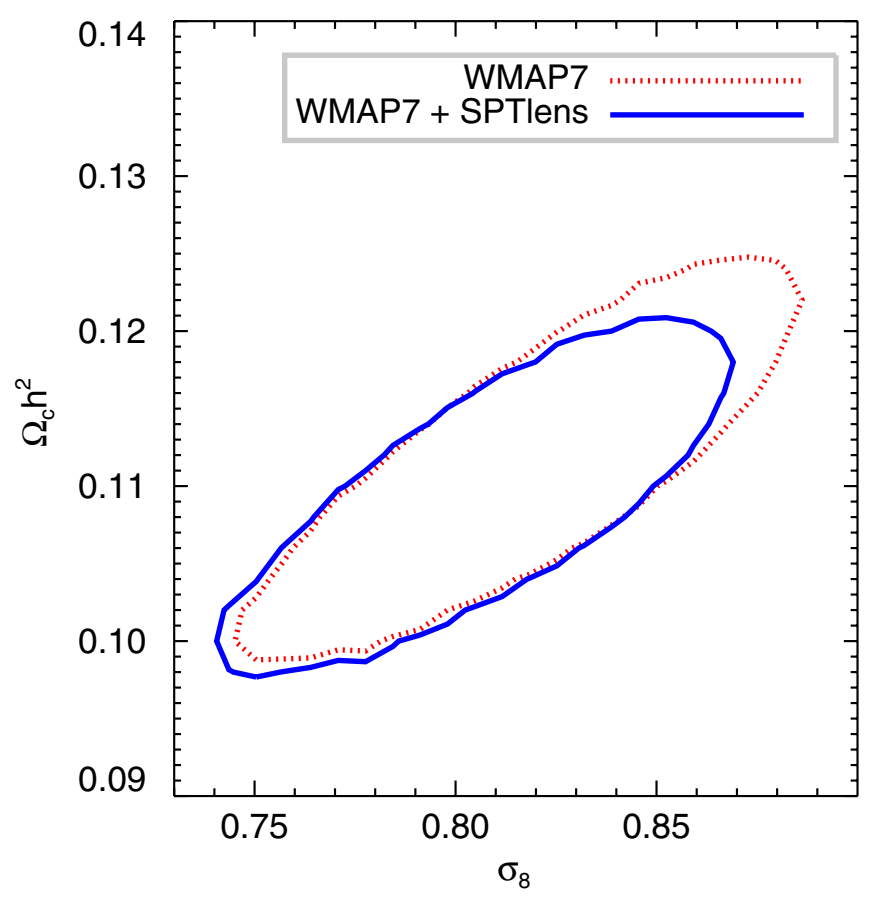

Figure 7. 95\% confidence-level constraints on $\sigma_{8}$ and $\Omega_{c} h^{2}$ from WMAP7 data alone (red dotted contour), and improvement when including the SPT lensing data (blue solid contour).

(A color version of this figure is available in the online journal.)

fluctuation power spectrum. In Figure 7, we show associated constraints on $\Omega_{c} h^{2}$ and $\sigma_{8}$, which is a derived quantity given by the square root of the variance of the linearly evolved density field today in spheres of size $8 \mathrm{~h}^{-1} \mathrm{Mpc}$. The constraint on $\sigma_{8}$ improves by $\sim 10 \%$, from $\sigma_{8}=0.821 \pm 0.029$ to $\sigma_{8}=0.810 \pm 0.026$ compared with WMAP7 alone.

O. Zahn et al. (2012, in preparation) suggest that for the SPT data considered here, the lensing information contained in 


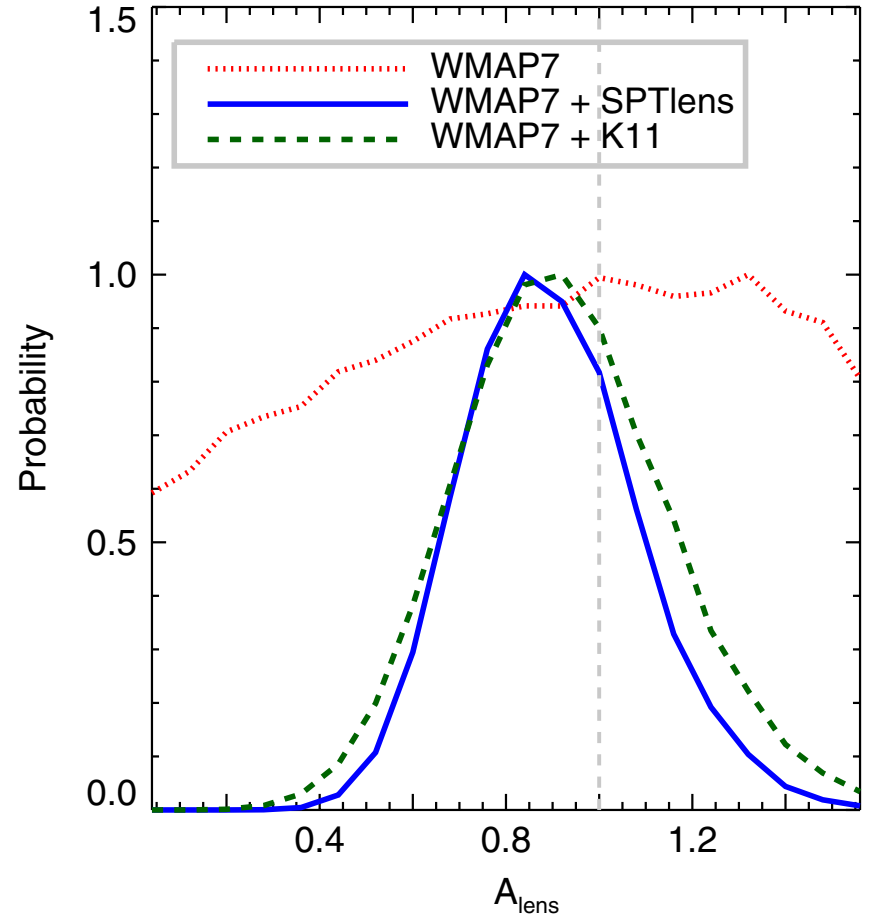

Figure 8. One-dimensional constraints on the lensing amplitude when marginalizing over $\Lambda$ CDM parameters. The WMAP power spectrum data (red dotted line) do not detect lensing. The SPT lensing data show a clear detection of lensing (solid blue curve), as do the SPT temperature power spectrum data from K11 (green dashed line).

(A color version of this figure is available in the online journal.)

CMB power spectrum and trispectrum estimates of the lensing amplitude are uncorrelated at the $\sim 90 \%$ level, apart from noise-induced correlations. ${ }^{32}$ Assuming the K11 temperature power spectrum data and lensing bandpowers derived in this work are independent, we obtain a constraint on the matter fluctuation amplitude of $\sigma_{8}=0.814 \pm 0.020$, an improvement in precision of $\sim 30 \%$ compared to WMAP7 alone.

$$
\text { 6.2.2. } A_{\text {lens }}
$$

In Section 6.1, we performed a fit for the lensing amplitude at a fixed reference cosmology. Here, we use MCMC techniques to find constraints on the lensing amplitude when marginalizing over $\Lambda \mathrm{CDM}$ parameters. At each point in the WMAP7 $\Lambda \mathrm{CDM}$ chain, we define a parameter, $A_{\text {lens }}$, which corresponds to the amplitude of the lensing power spectrum relative to its value for the given set of $\Lambda \mathrm{CDM}$ cosmological parameters. We can then find constraints on this parameter, to which we assign a flat prior, jointly with the $\Lambda \mathrm{CDM}$ parameters. K11 used this approach to measure the lensing amplitude at high significance. We find that the SPT lensing data in combination with WMAP7 measure the lensing amplitude to be $A_{\text {lens }}=0.90 \pm 0.19$. The equivalent measure of the lensing impact on the temperature power spectrum from $\mathrm{K} 11$ is $A_{\text {lens }}=0.92 \pm 0.23$. These constraints are shown in Figure 8. Combining the SPT lensing data with $\mathrm{K} 11$, neglecting any possible correlation between the lensing information, gives $A_{\text {lens }}=0.90 \pm 0.15$, with the six $\Lambda \mathrm{CDM}$ parameters marginalized.

\footnotetext{
32 Reducing the noise correlations through the method proposed in Hanson et al. (2011), which we have not done in this work, increases the sensitivity of the trispectrum-based estimate of the lensing amplitude without affecting the power-spectrum-based estimate, making our simplified combination conservative given the disclaimer of the $\sim 10 \%$ level correlation.
}

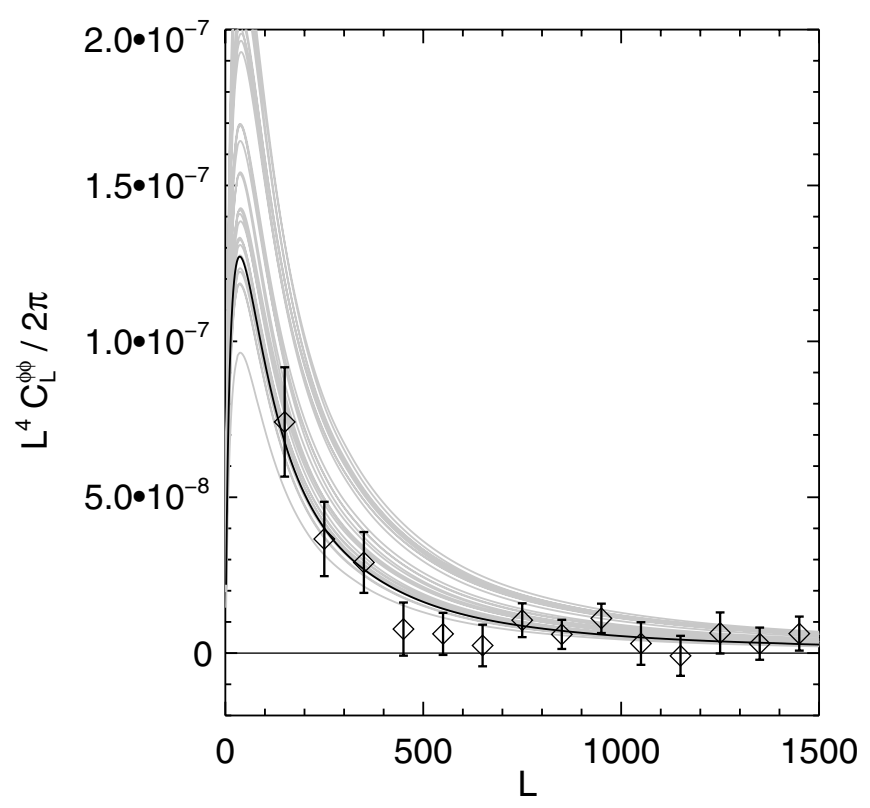

Figure 9. Demonstrating the ability of the lensing data to constrain the freecurvature model. Each gray line is the lensing power spectrum for a point in cosmological parameter space allowed by WMAP7 when the curvature parameter is allowed to vary. Specifically, the curves are taken from equally spaced points in a WMAP7-only Markov chain which explores the $\Lambda$ CDM $+\Omega_{k}$ model. The SPT lensing bandpowers, shown as black diamonds, can significantly discriminate among the models. The lensing power spectrum for the fiducial cosmology, which has $\Omega_{k}=0$, is shown as the black curve.

\subsubsection{Curvature}

Observations of the primary CMB at $z \sim 1100$ do not measure the spatial curvature of the universe to high precision. This is due to the angular diameter distance degeneracy. A key physical length scale associated with the observed CMB surface is the acoustic scale, and observations of the CMB that include the acoustic peak region of the power spectrum can measure the angular size corresponding to this physical scale to high accuracy. Indeed, the parameter $\theta_{s}$ is used as one of the standard base parameters in cosmological fitting. There is an effective degree of freedom associated with the angular diameter distance to the $\mathrm{CMB}$ recombination surface, which is required to convert the angular size to a physical length scale. In the flat $\Lambda$ CDM model (as we parameterize it), $\Omega_{\Lambda}$ plays this role, and is well constrained from primary $\mathrm{CMB}$ data (to $\sim 4 \%$ ), despite the fact that the dynamical effects of dark energy become important long after last scattering.

However, in cosmological models which allow for an additional free parameter that affects the angular size of the sound horizon, such as curvature, only a particular linear combination of these parameters will be well constrained with primary CMB data, leading to a strong parameter degeneracy. Adding a measurement of the distance scale to another redshift range, such as that containing the matter fluctuations responsible for CMB lensing, can break this degeneracy (e.g., Smith 2006) as was shown experimentally by Sherwin et al. (2011). Indeed, Figure 9 demonstrates that models with negative curvature, which are allowed by primary CMB temperature measurements, can predict CMB lensing potential power spectra that are up to a factor of two higher in amplitude than those predicted by $\Lambda \mathrm{CDM}$ in flat geometries.

Here, we evaluate the improvement in the measure of the curvature parameter $\Omega_{k}$ when the SPT lensing data are considered 

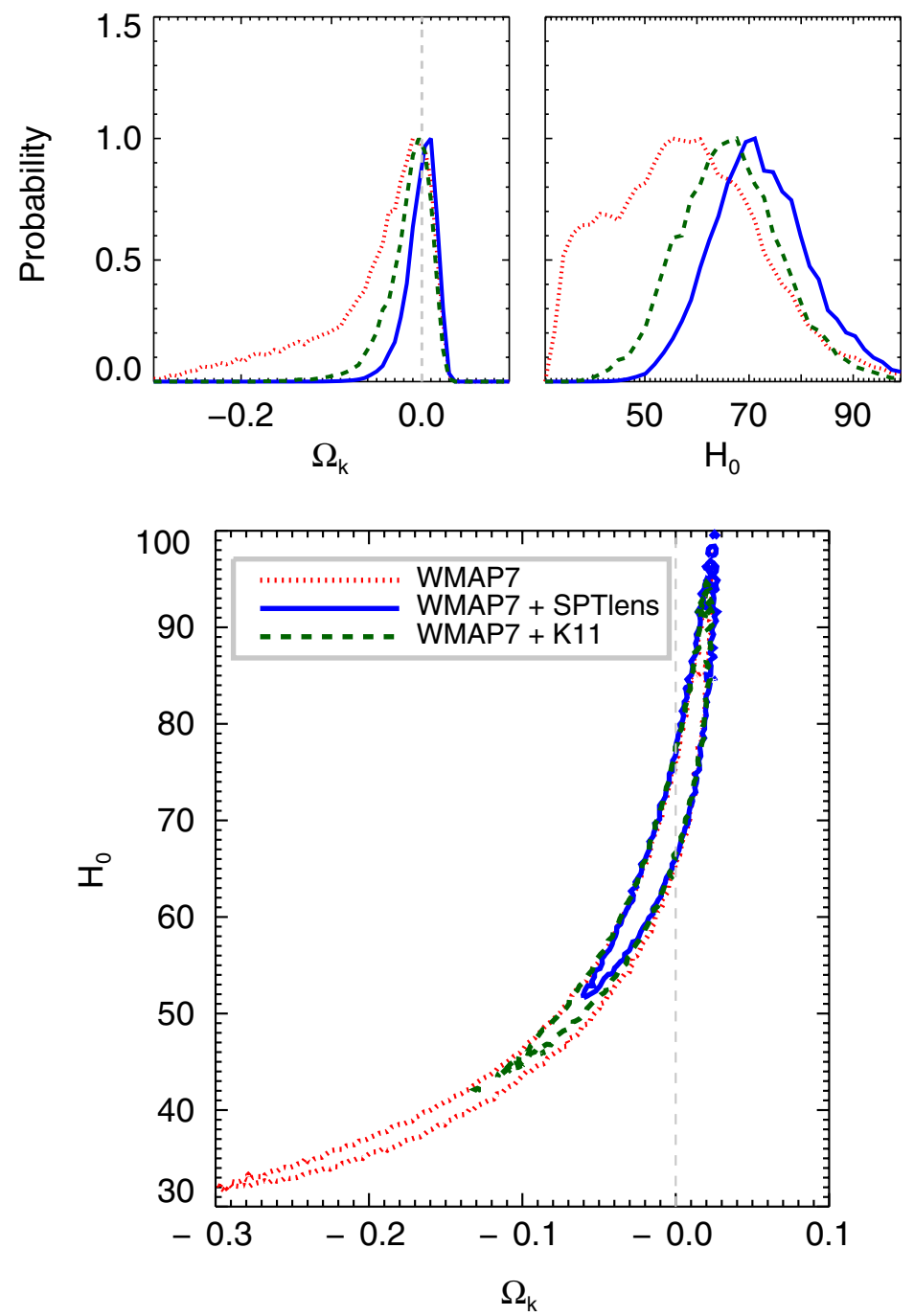
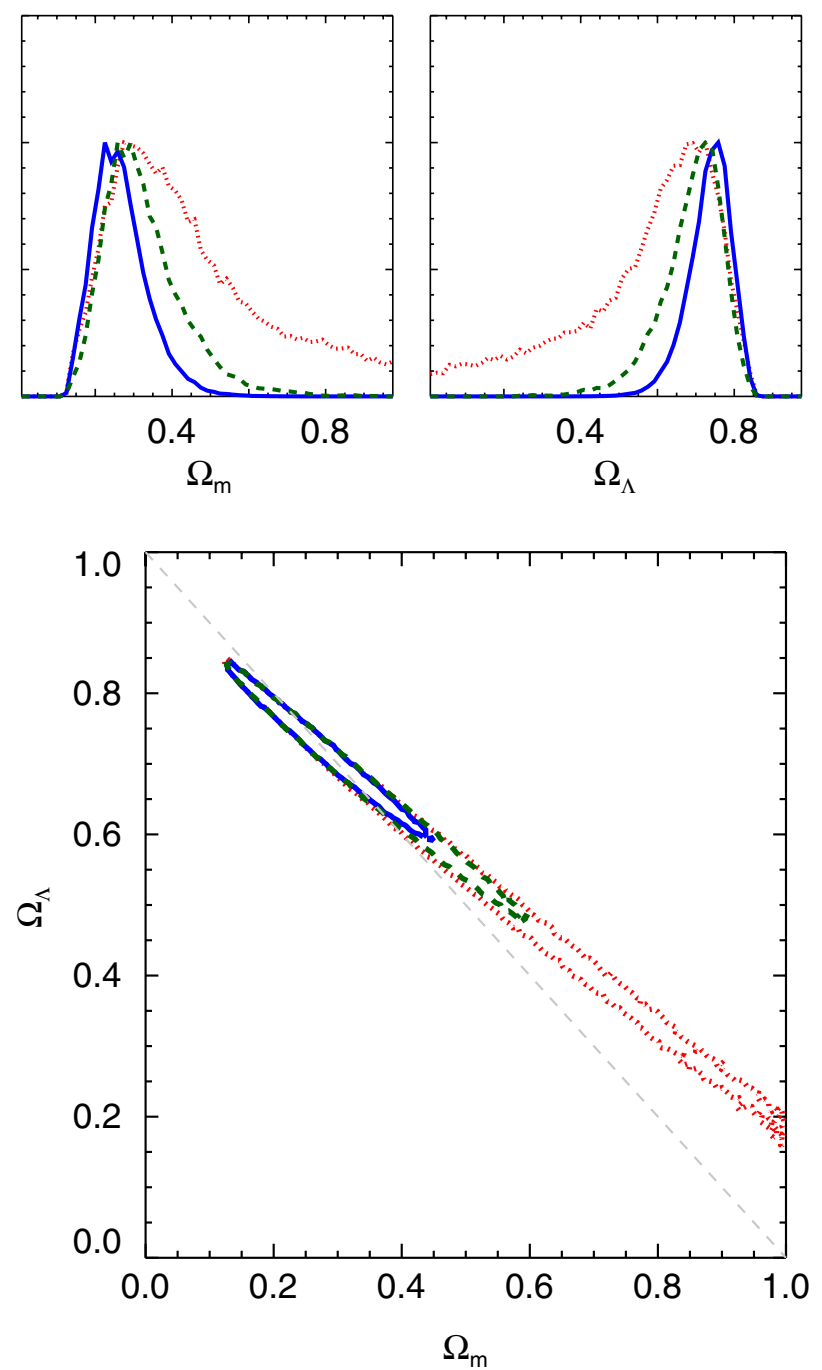

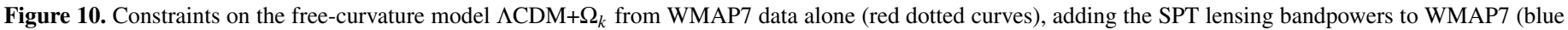

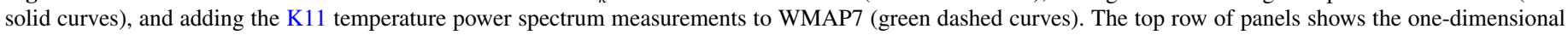
parameter likelihoods on $\Omega_{k}, H_{0}, \Omega_{m}$, and $\Omega_{\Lambda}$; the two panels on the bottom show the $95 \%$ confidence-level contours in the $H_{0}-\Omega_{k}$ and $\Omega_{\Lambda}-\Omega_{m}$ planes.

(A color version of this figure is available in the online journal.)

in combination with the WMAP7 data, by adding the constraints from the SPT lensing bandpowers to the WMAP7-allowed $\Lambda \mathrm{CDM}+\Omega_{k}$ parameter volume. We find the marginalized $1 \sigma$ curvature constraint to tighten by a factor of $\sim 3.9$ over WMAP7 alone, to $\Omega_{k}=-0.001 \pm 0.017$. Many of the models allowed by WMAP7 correspond to values of the Hubble parameter $H_{0}$ as low as $30 \mathrm{~km} \mathrm{~s}^{-1} \mathrm{Mpc}^{-1}$; adding the SPT lensing data leads to an effective measure of $H_{0}=(72.3 \pm 9.3) \mathrm{km} \mathrm{s}^{-1} \mathrm{Mpc}^{-1}$, from the CMB alone. This result is not currently competitive with direct measures of the Hubble constant, which have an uncertainty of $2.4 \mathrm{~km} \mathrm{~s}^{-1} \mathrm{Mpc}^{-1}$ (Riess et al. 2011), but is of interest because the constraints come only from the CMB. These results are shown in Figure 10. The constraint from the CMB lensing measurement also corresponds to a measure of a nonzero dark energy density, using only the CMB, of $\Omega_{\Lambda}=0.734 \pm 0.056$. Such a measure is not possible using only the primary CMB anisotropies at recombination, without additional information from lensing.

The greater-than- $5 \sigma$ constraint of the lensing amplitude found using the lensing effect on the CMB power spectrum by $\mathrm{K} 11$ (when accounting for the non-Gaussian probability distribution in $A_{\text {lens }}$ ) is also able to provide significant constraints on this parameter volume. This corresponds to a measurement of the curvature of the universe using only the CMB power spectrum, i.e., without performing the trispectrum-based lensing reconstruction that is the focus of this paper. As is shown with the green dashed curves in Figure 10, the K11 temperature bandpowers, together with WMAP7, constrain the dark energy density to $\Omega_{\Lambda}=0.689 \pm 0.081$, the Hubble parameter to $H_{0}=66.4 \pm 9.8 \mathrm{~km} \mathrm{~s}^{-1} \mathrm{Mpc}^{-1}$, and the curvature parameter to $\Omega_{k}=-0.015 \pm 0.026$. We have checked that these results are almost entirely due to the lensing effect on the K11 temperature power spectrum measurements; the constraints on these parameters degrade to close to their WMAP7-alone values when the $A_{\text {lens }}$ parameter is marginalized. Measures of the dark energy from the CMB alone are thus possible without performing lensing reconstruction, using only the effects of lensing on the CMB temperature power spectrum.

\subsubsection{Neutrino Masses}

Massive neutrinos damp the matter power spectrum on scales which are smaller than their free-streaming scale at the redshift 


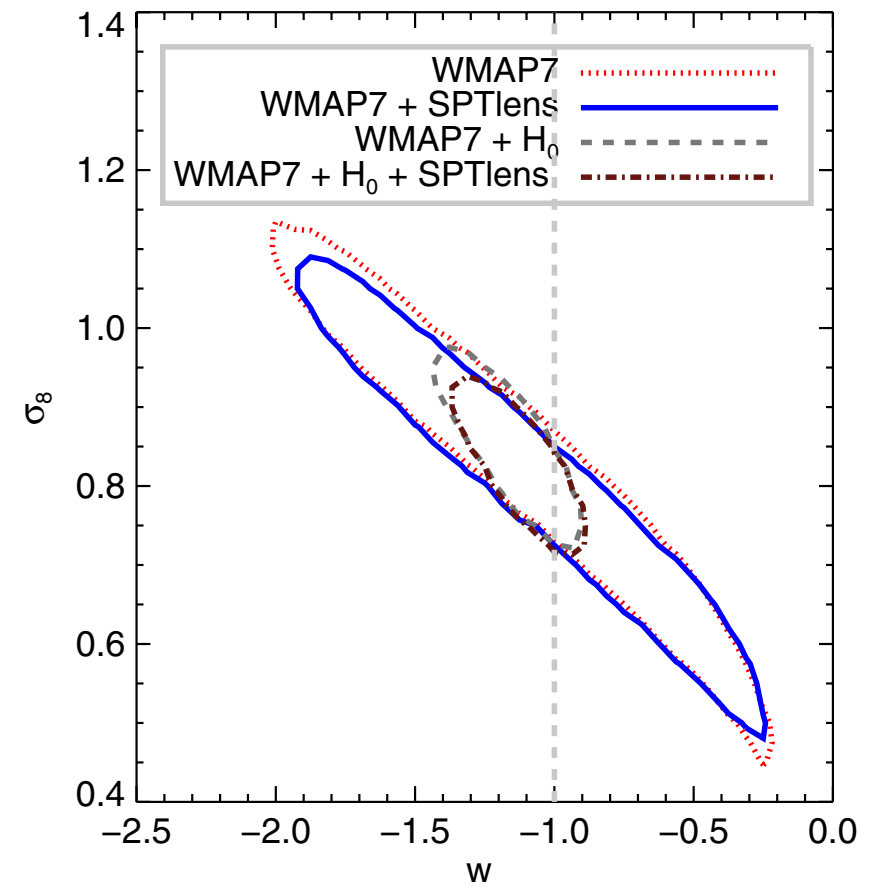

Figure 11.95\% confidence-level contours on the dark energy equation of state $w$ and the matter fluctuation amplitude $\sigma_{8}$. The WMAP7 data (red dotted contour) show a strong degeneracy between these two parameters. Adding the SPT lensing data (blue solid contour) can start to break this degeneracy, tightening the $w$ constraint by $5 \%$. When the measure of the Hubble parameter is also used (gray dashed contour), the lensing data improve the constraint by $15 \%$ (brown dot-dashed contour).

(A color version of this figure is available in the online journal.)

at which they become non-relativistic. For the case of three neutrinos with degenerate masses, an increase in the sum of the neutrino masses of $0.1 \mathrm{eV}$ leads to a decrease of $5 \%$ in the matter power spectrum on scales of $k \gtrsim 0.05 h \mathrm{Mpc}^{-1}$. The matter power spectrum suppression leads to a comparable level of suppression in the CMB lensing power spectrum at $L \gtrsim 100$ (Kaplinghat et al. 2003; Lesgourgues et al. 2006), corresponding to the entire SPT signal band. High-significance CMB lensing measurements hold the promise to measure the sum of neutrino masses at the $0.05 \mathrm{eV}$ level (e.g., Lesgourgues et al. 2006; de Putter et al. 2009), the minimum required for at least one species by oscillation experiments (Adamson et al. 2009).

We generate lensing power spectra from the WMAP7-allowed $\Lambda \mathrm{CDM}+\Sigma m_{v}$ parameter space and again compute SPT lensing likelihoods for each model. Although the SPT lensing data have the statistical power to improve the constraint on the sum of neutrino masses by $\sim 20 \%$, they also show a mild preference for low values of both $\sigma_{8}$ and $\Omega_{c} h^{2}$, as seen (in the case of $\Lambda \mathrm{CDM}$ ) in Figure 7 . Both of these parameters are degenerate with the neutrino masses. The mild preference for low values of $\sigma_{8}$ corresponds to a mild preference for larger values of $\Sigma m_{v}$. The net result is that the WMAP7-based $95 \%$ confidence-level upper limit of the sum of neutrino masses actually increases slightly, from $\Sigma m_{v}<1.10 \mathrm{eV}$ to $\Sigma m_{v}<1.17 \mathrm{eV}$.

A significant fraction of the parameter space allowed by WMAP7 corresponds to values of the Hubble parameter which are inconsistent with recent observations. With the measure of the Hubble parameter of Riess et al. (2011) included with WMAP7, adding the SPT lensing data changes the $95 \%$ confidence-level upper limit from $\Sigma m_{v}<0.36 \mathrm{eV}$ to $\Sigma m_{v}<$ $0.38 \mathrm{eV}$.

\subsubsection{Dark Energy Equation of State}

The majority of the weight in the redshift kernel for CMB lensing, Equation (1), lies in the matter-dominated era. The amplitude of the lensing power spectrum can thus be used to provide a measure of the distance to these redshifts, leading to constraints on the equation of state of dark energy, $w$. Assuming $w$ to be constant as a function of redshift, we show the constraints in the $w-\sigma_{8}$ plane in Figure 11. WMAP7 weakly constrains $w$, to $-1.120 \pm 0.420$, based on the measure of the expansion history provided by CMB observations. Adding the SPT lensing data modestly improves this uncertainty on $w$, by $5 \%$.

When including the Riess et al. (2011) $H_{0}$ measurement, together with WMAP7, the SPT lensing data improve the precision of $w$ by $15 \%$, from $w=-1.126 \pm 0.111$ to $w=$ $-1.087 \pm 0.096$.

\section{CONCLUSIONS}

We have detected the power spectrum of gravitational lensing of the $\mathrm{CMB}$ at high significance on scales of $8^{\prime}$ and larger $(L<1500)$. We find the amplitude of the measured signal in our fiducial best-fit $\Lambda \mathrm{CDM}$ cosmology to be $A_{\text {lens }}^{0}=0.86 \pm 0.16$. This detection represents an important step toward the eventual goal of using the lensing of the microwave background as a precise probe of the growth of structure and geometry of the universe.

As part of this analysis, we have modeled several important biases in lensing reconstruction, demonstrating the ability to remove the leading bias due to the Gaussian power in the map. We have used two complementary approaches for dealing with this bias. In the first approach, we estimate the bias directly, relying heavily on previous SPT results: the measured power spectrum of the primary CMB (K11), backgrounds from dusty galaxies and galaxy clusters (Lueker et al. 2010; Shirokoff et al. 2011; Reichardt et al. 2011), and the known source counts in the maps (Vieira et al. 2010). In principle, this method leads to the maximum possible detection significance, as it uses all of the available data. However, since the Gaussian bias exceeds the signal by a large factor over much of the lensing signal band, uncertainties due to the instrumental calibration and beam or small uncertainties in power spectrum estimation lead to systematic uncertainty. We obtain a $6.3 \sigma$ detection of CMB lensing while accounting for these sources of systematic uncertainty.

In the second approach, lensing maps obtained from two disjoint regions of Fourier space were cross-correlated. This ensured that there was no Gaussian bias to remove (Hu 2001b; Sherwin \& Das 2010). The clear SPT detection of this signal provided a more direct indication of lensing of the CMB. This method is more robust to systematics, but has less statistical significance (as implemented), providing a $3.9 \sigma$ detection. However, the loss in signal-to-noise ratio is not a fundamental property of the lensing measurement using this method. More sophisticated techniques for dividing the Fourier domain into several regions and combining the multiple quadratic pairings of these regions should lead to an increased signal-to-noise ratio. Without a large (and somewhat uncertain) noise bias to subtract, this is a potentially cleaner signal for future measurements; experiments with higher signal-to-noise ratio will require stricter control of systematic uncertainties to subtract the noise bias to substantially higher precision.

We have also extracted an estimate of the power spectrum of a curl-like component in the lensing field. This is a strong test of 
our ability to measure the noise bias, since similar forms for the noise bias appear in both the divergence and curl estimates. By detecting the curl-like component at the expected level, we have passed a significant test of our understanding of the Gaussian backgrounds and noise in the SPT experiment. Furthermore, we have obtained $1.8 \sigma$ evidence of the lensing signal using this curl estimator by itself: while the curl estimator is formulated to reconstruct fields with the opposite parity than leading-order lensing, higher-order lensing biases, similar to those found in Kesden et al. (2003), lead to a nonzero lensing signal in the curl estimate.

The contamination of the lensing signal by non-Gaussianity in Galactic and extragalactic foregrounds was simulated and found to be relatively small. The ability of SPT to detect point sources down to relatively low flux levels has made it possible to mask out point sources to a level where the point-source background becomes nearly Gaussian. There is a residual bias originating from the correlations between the point-source field and the lensing field; although in the current work it was neglected given our $15 \%$ statistical uncertainty, this bias will need to be better understood in future analyses. In the case of SZ emission from galaxy clusters, the lower-than-expected SZ signal measured by Lueker et al. (2010), Das et al. (2011a), Shirokoff et al. (2011), Dunkley et al. (2011), and Reichardt et al. (2011) means that only a handful of clusters need to be masked to reduce the SZ contamination to a level that can be neglected for this analysis.

We have also investigated the constraints that our measurement of the lensing power spectrum places on cosmological models. We found that adding our measurement to those from WMAP7 improved the precision of the measurement of the amplitude of matter density fluctuations, $\sigma_{8}$, by $10 \%$. The lensing amplitude, marginalized over WMAP7-allowed models, was found to be $A_{\text {lens }}=0.90 \pm 0.19$. The lensing data are able to mildly break degeneracies in parameter values that result from the analysis of primary CMB data, namely, $w$ and $\Sigma m_{v}$. When also including external measures of the Hubble parameter, the constraint on $w$ improved by $15 \%$ when including the SPT lensing data, to $w=-1.087 \pm 0.096$. Additionally, as in Sherwin et al. (2011), we found that our measurement can break the angular diameter distance degeneracy and constrain models with spatial curvature. We found $\sigma\left(\Omega_{k}\right)=0.017$ when combining with WMAP7, and $\sigma\left(\Omega_{k}\right) \simeq 0.015$ when including the lensing effect on the SPT CMB temperature power spectrum reported by K11.

Measurements of $\mathrm{CMB}$ lensing are expected to continue to rapidly improve. The recently completed full SPT-SZ survey includes approximately $2500 \mathrm{deg}^{2}$ of CMB temperature measurements at the same depth as those considered here, along with additional measurements at $95 \mathrm{GHz}$ and $220 \mathrm{GHz}$. This survey should produce a detection of the lensing signal at several times the significance of the detection presented here.

The analysis of the full SPT-SZ survey will require a more careful modeling of foreground astrophysics than we have performed here; for this analysis, we only included modes with $l<3000$ in the CMB maps to avoid contamination by galaxies and galaxy clusters. However, there is signal on smaller scales that can be recovered with a more careful treatment of non-Gaussianity from foregrounds. Upcoming polarizationsensitive CMB experiments (e.g., SPTpol, McMahon et al. 2009; ACTpol, Niemack et al. 2010; PolarBear, Arnold et al. 2010) will reconstruct the lensing power spectrum with high signal-tonoise ratio, but will need to deal with a distinct set of systematic uncertainties (Su et al. 2009; Miller et al. 2009). The robust detection presented here and the parameter constraints that are enabled indicate that $\mathrm{CMB}$ lensing is emerging as a powerful probe of cosmology.

We thank E. Anderes, S. Das, S. Dodelson, O. Doré, D. Hanson, W. Hu, and B. Sherwin for useful discussions.

The South Pole Telescope is supported by the National Science Foundation through grants ANT-0638937 and ANT0130612. Partial support is also provided by the NSF Physics Frontier Center grant PHY-0114422 to the Kavli Institute of Cosmological Physics at the University of Chicago, the Kavli Foundation and the Gordon and Betty Moore Foundation. The McGill group acknowledges funding from the National Sciences and Engineering Research Council of Canada, Canada Research Chairs program, and the Canadian Institute for Advanced Research. Oliver Zahn acknowledges support from an Inaugural Berkeley Center for Cosmological Physics Fellowship. R. Keisler acknowledges support from the NASA Hubble Fellowship grant HF-51275.01. B.A. Benson is supported by a KICP Fellowship. M. Dobbs acknowledges support from an Alfred P. Sloan Research Fellowship. L. Shaw acknowledges the support of Yale University and the NSF grant AST-1009811. M. Millea and L. Knox acknowledge the support of the NSF grant 0709498. J. Mohr acknowledges support from the Excellence Cluster Universe and the DFG research program TR33 "Dark Universe." This research used resources of the National Energy Research Scientific Computing Center, which is supported by the Office of Science of the U.S. Department of Energy under Contract No. DE-AC02-05CH11231. It also used resources of the CLUMEQ supercomputing consortium, part of the Compute Canada network. We acknowledge the use of the Legacy Archive for Microwave Background Data Analysis (LAMBDA). Support for LAMBDA is provided by the NASA Office of Space Science. Some of the results in this paper have been derived using the HEALPix (Górski et al. 2005) package.

\section{APPENDIX}

\section{LENSING SIGNATURE IN THE CURL ESTIMATOR POWER SPECTRUM}

As discussed in Section 4.1.1, the curl estimator of Cooray et al. (2005) is formulated to search for curl-like sources of deflection in the CMB. Instead of shifting the CMB by the gradient of a scalar field $\phi$ according the usual lensing operation $T(\hat{\mathbf{n}})=T^{U}(\hat{\mathbf{n}}+\nabla \phi(\hat{\mathbf{n}}))$, these sources, denoted $\Omega(\hat{\mathbf{n}})$, shift the CMB according to

$$
T(\hat{\mathbf{n}})=T^{U}(\hat{\mathbf{n}}+\nabla \star \Omega(\hat{\mathbf{n}})) .
$$

The operator $\star$ is given by $\mathbf{A} \star \mathbf{B}=A_{y} B_{x}-A_{x} B_{y}$. The signature of this mode of deflection is negligibly small in a given reconstructed $\phi$ map. However, additional terms in the lensing trispectrum lead to the bias $N_{L}^{(1)}$ in the estimated power spectrum of this map, as they do for the divergence estimator of Kesden et al. (2003). Indeed, in the main text we show that evidence for this signal in the curl estimator is seen in the SPT data at $1.8 \sigma$. In Figure 12, we show the prediction, for both the divergence and curl components, under the assumptions of case of isotropic white noise and analytical beams. In practice, however, when presenting our results we compare against the prediction for Monte Carlo estimates, in order to take into account the anisotropic noise properties of the real data set. 

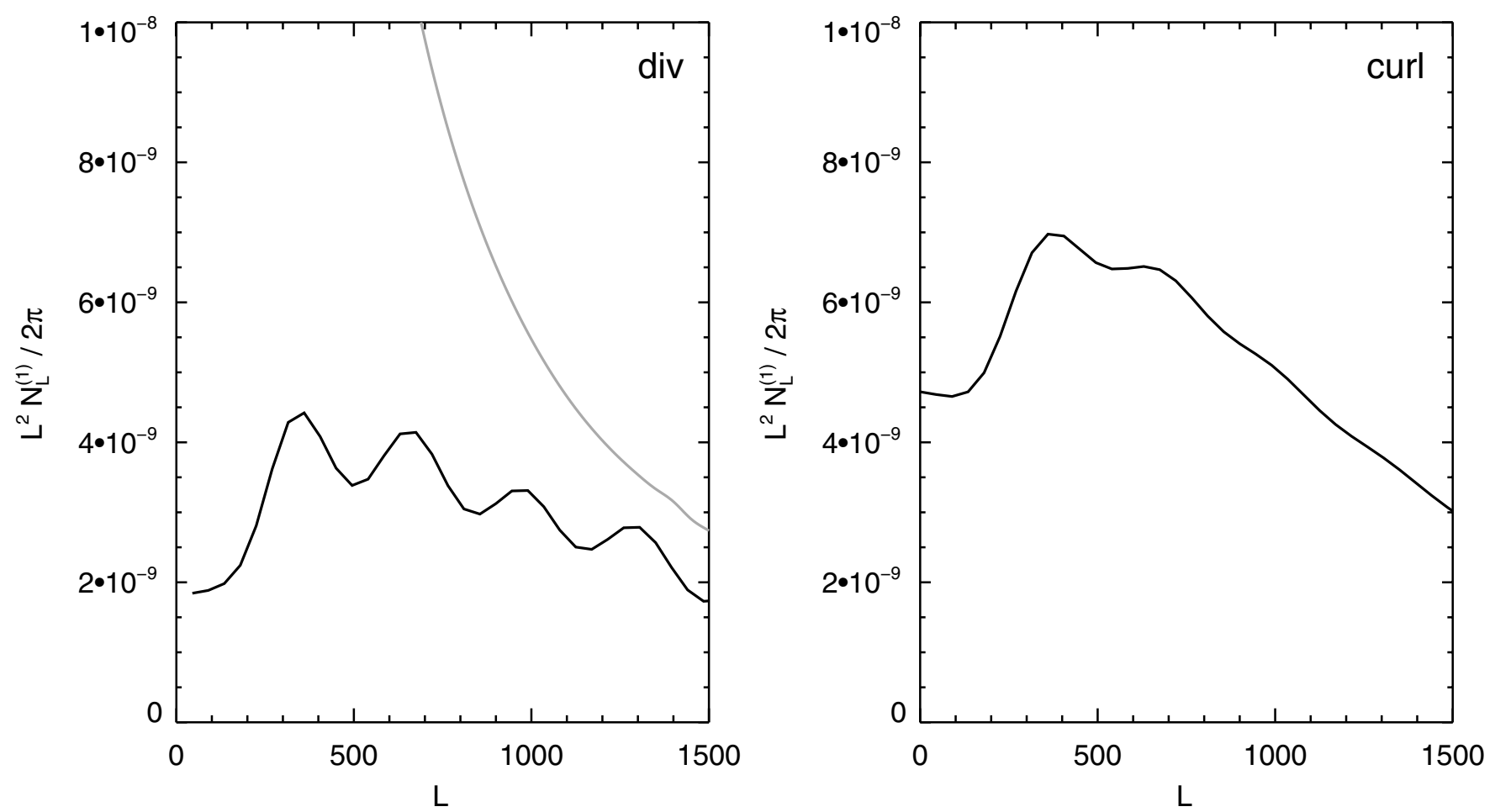

Figure 12. Higher-order bias signatures in the lensing estimate, described further in Section 4.3.3. Left: Kesden et al. (2003) bias in the divergence estimate (black) together with the lensing power spectrum (gray). Right: similar bias in the curl estimate. These are computed under the assumption of isotropic white noise and an analytical beam.

\section{REFERENCES}

Adamson, P., Aguilar-Arevalo, A. A., Anderson, C. E., et al. 2009, Phys. Rev. Lett., 102, 211801

Addison, G. E., Dunkley, J., Hajian, A., et al. 2012, ApJ, 752, 120

Amblard, A., Vale, C., \& White, M. 2004, New Astron., 9, 687

Anderes, E., Knox, L., \& van Engelen, A. 2011, Phys. Rev. D, 83, 043523

Arnold, K., Ade, P. A. R., Anthony, A. E., et al. 2010, Proc. SPIE, 7741, 77411E

Benson, B. A., de Haan, T., Dudley, J. P., et al. 2011, arXiv:1112.5435

Bernardeau, F. 1997, A\&A, 324, 15

Blanchard, A., \& Schneider, J. 1987, A\&A, 184, 1

Bond, J. R., Jaffe, A. H., \& Knox, L. 2000, ApJ, 533, 19

Brown, M. L., Ade, P., Bock, J., et al. 2009, ApJ, 705, 978

Bucher, M., Sofia Carvalho, C., Moodley, K., \& Remazeilles, M. 2012, Phys. Rev. D, 85, 043016

Calabrese, E., Cooray, A., Martinelli, M., et al. 2009, Phys. Rev. D, 80, 103516

Calabrese, E., Slosar, A., Melchiorri, A., Smoot, G. F., \& Zahn, O. 2008, Phys. Rev. D, 77, 123531

Carlstrom, J. E., Ade, P. A. R., Aird, K. A., et al. 2011, PASP, 123, 568

Carvalho, C. S., \& Moodley, K. 2010, Phys. Rev. D, 81, 123010

Christensen, N., Meyer, R., Knox, L., \& Luey, B. 2001, Class. Quantum Grav., 18,2677

Cole, S., \& Kaiser, N. 1989, MNRAS, 237, 1127

Cooray, A., \& Hu, W. 2000, ApJ, 534, 533

Cooray, A., Kamionkowski, M., \& Caldwell, R. R. 2005, Phys. Rev. D, 71,123527

Cooray, A., \& Kesden, M. 2003, New Astron., 8, 231

Das, S., Marriage, T. A., Ade, P. A. R., et al. 2011a, ApJ, 729, 62

Das, S., Sherwin, B. D., Aguirre, P., et al. 2011b, Phys. Rev. Lett., 107, 021301 de Putter, R., Zahn, O., \& Linder, E. V. 2009, Phys. Rev. D, 79, 065033

de Zotti, G., Ricci, R., Mesa, D., et al. 2005, A\&A, 431, 893

Dunkley, J., Hlozek, R., Sievers, J., et al. 2011, ApJ, 739, 52

Feng, C., Keating, B., Paar, H. P., \& Zahn, O. 2012, Phys. Rev. D, 85, 043513

Finkbeiner, D. P., Davis, M., \& Schlegel, D. J. 1999, ApJ, 524, 867

Foley, R. J., Andersson, K., Bazin, G., et al. 2011, ApJ, 731, 86

Fosalba, P., Gaztañaga, E., \& Castander, F. J. 2003, ApJ, 597, L89

Fowler, J. W., Acquaviva, V., Ade, P. A. R., et al. 2010, ApJ, 722, 1148

Friedman, R. B., Ade, P., Bock, J., et al. 2009, ApJ, 700, L187

Górski, K. M., Hivon, E., Banday, A. J., et al. 2005, ApJ, 622, 759

Gottlöber, S., \& Yepes, G. 2007, ApJ, 664, 117

Hajian, A., Viero, M. P., Addison, G., et al. 2012, ApJ, 744, 40
Hall, N. R., Keisler, R., Knox, L., et al. 2010, ApJ, 718, 632

Hanson, D., Challinor, A., Efstathiou, G., \& Bielewicz, P. 2011, Phys. Rev. D, 83,043005

Hirata, C. M., Ho, S., Padmanabhan, N., Seljak, U., \& Bahcall, N. A. 2008, Phys. Rev. D, 78, 043520

Hirata, C. M., \& Seljak, U. 2003a, Phys. Rev. D, 67, 043001

Hirata, C. M., \& Seljak, U. 2003b, Phys. Rev. D, 68, 083002

Hoffman, Y., \& Ribak, E. 1991, ApJ, 380, L5

Hu, W. 2001a, Phys. Rev. D, 64, 083005

Hu, W. 2001b, ApJ, 557, L79

Hu, W., DeDeo, S., \& Vale, C. 2007, New J. Phys., 9, 441

Hu, W., \& Okamoto, T. 2002, ApJ, 574, 566

Jarosik, N., Bennett, C. L., Dunkley, J., et al. 2011, ApJS, 192, 14

Kaplinghat, M., Knox, L., \& Song, Y.-S. 2003, Phys. Rev. Lett., 91, 241301

Keisler, R., Reichardt, C. L., Aird, K. A., et al. 2011, ApJ, 743, 28

Kesden, M., Cooray, A., \& Kamionkowski, M. 2003, Phys. Rev. D, 67, 123507

Knox, L., Bond, J. R., Jaffe, A. H., Segal, M., \& Charbonneau, D. 1998, Phys. Rev. D, 58, 083004

Kogut, A., Spergel, D. N., Barnes, C., et al. 2003, ApJS, 148, 161

Komatsu, E., Smith, K. M., Dunkley, J., et al. 2011, ApJS, 192, 18

Lesgourgues, J., Liguori, M., Matarrese, S., \& Riotto, A. 2005, Phys. Rev. D, 71,103514

Lesgourgues, J., Perotto, L., Pastor, S., \& Piat, M. 2006, Phys. Rev. D, 73, 045021

Lewis, A. 2005, Phys. Rev. D, 71, 083008

Lewis, A., \& Bridle, S. 2002, Phys. Rev. D, 66, 103511

Lewis, A., \& Challinor, A. 2006, Phys. Rep., 429, 1

Lewis, A., Challinor, A., \& Lasenby, A. 2000, ApJ, 538, 473

Lueker, M., Reichardt, C. L., Schaffer, K. K., et al. 2010, ApJ, 719, 1045

Marriage, T. A., Baptiste Juin, J., Lin, Y.-T., et al. 2011, ApJ, 731, 100

McMahon, J. J., Aird, K. A., Benson, B. A., et al. 2009, in AIP Conf. Ser. 1185,

The Thirteenth International Workshop on Low Temperature Detectors, ed. B. Young, B. Cabrera, \& A. Miller (Melville, NY: AIP), 511

Metropolis, N., Rosenbluth, A. W., Rosenbluth, M. N., Teller, A. H., \& Teller, E. 1953, J. Chem. Phys., 21, 1087

Miller, N. J., Shimon, M., \& Keating, B. G. 2009, Phys. Rev. D, 79, 063008

Negrello, M., Perrotta, F., González-Nuevo, J., et al. 2007, MNRAS, 377, 1557

Niemack, M. D., Ade, P. A. R., Aguirre, J., et al. 2010, Proc. SPIE, 7741, 77411 S

Okamoto, T., \& Hu, W. 2003, Phys. Rev. D, 67, 083002

Padin, S., Staniszewski, Z., Keisler, R., et al. 2008, Appl. Opt., 47, 4418 
Padmanabhan, N., Hirata, C. M., Seljak, U., et al. 2005, Phys. Rev. D, 72, 043525

Perotto, L., Bobin, J., Plaszczynski, S., Starck, J., \& Lavabre, A. 2010, A\&A, 519, A4

Planck Collaboration, Ade, P. A. R., Aghanim, N., et al. 2011a, A\&A, 536, A13 Planck Collaboration, Ade, P. A. R., Aghanim, N., et al. 2011b, A\&A, 536, A18

Reichardt, C. L., Ade, P. A. R., Bock, J. J., et al. 2009, ApJ, 694, 1200

Reichardt, C. L., Shaw, L. D., Zahn, O., et al. 2011, ApJ, in press

Riess, A. G., Macri, L., Casertano, S., et al. 2011, ApJ, 730, 119

Ruhl, J., Ade, P. A. R., Carlstrom, J. E., et al. 2004, Proc. SPIE, 5498, 11

Sehgal, N., Bode, P., Das, S., et al. 2010, ApJ, 709, 920

Sehgal, N., Trac, H., Acquaviva, V., et al. 2011, ApJ, 732, 44

Seljak, U. 1996, ApJ, 463, 1

Seljak, U., \& Zaldarriaga, M. 1999, Phys. Rev. Lett., 82, 2636

Sherwin, B. D., \& Das, S. 2010, arXiv:1011.4510

Sherwin, B. D., Dunkley, J., Das, S., et al. 2011, Phys. Rev. Lett., 107, 021302

Shirokoff, E., Reichardt, C. L., Shaw, L., et al. 2011, ApJ, 736, 61
Smidt, J., Cooray, A., Amblard, A., et al. 2011, ApJ, 728, L1

Smith, K. M. 2006, Phys. Rev. D, 74, 083002

Smith, K. M., Hu, W., \& Kaplinghat, M. 2006, Phys. Rev. D, 74, 123002

Smith, K. M., Zahn, O., \& Doré, O. 2007, Phys. Rev. D, 76, 043510

Snyder, J. P. 1987, Map Projections-A Working Manual (Washington, DC: U.S. Geological Survey)

Song, Y.-S., Cooray, A., Knox, L., \& Zaldarriaga, M. 2003, ApJ, 590, 664

Staniszewski, Z., Ade, P. A. R., Aird, K. A., et al. 2009, ApJ, 701, 32

Su, M., Yadav, A. P. S., \& Zaldarriaga, M. 2009, Phys. Rev. D, 79, 123002

Sunyaev, R. A., \& Zel'dovich, Y. B. 1972, Comments Astrophys. Space Phys., 4, 173

Vanderlinde, K., Crawford, T. M., de Haan, T., et al. 2010, ApJ, 722, 1180

Vieira, J. D., Crawford, T. M., Switzer, E. R., et al. 2010, ApJ, 719, 763

Williamson, R., Benson, B. A., High, F. W., et al. 2011, ApJ, 738, 139

Zahn, O., Reichardt, C. L., Shaw, L., et al. 2011, arXiv:1111.6386

Zahn, O., \& Zaldarriaga, M. 2006, ApJ, 653, 922

Zaldarriaga, M. 2000, Phys. Rev. D, 62, 063510

Zaldarriaga, M., \& Seljak, U. 1999, Phys. Rev. D, 59, 123507 